\title{
The Monoid Consisting of Kuratowski Operations
}

\author{
Szymon Plewik and Marta Walczyńska \\ Institute of Mathematics, University of Silesia, ul. Bankowa 14, 40-007 Katowice, Poland \\ Correspondence should be addressed to Szymon Plewik; plewik@math.us.edu.pl
}

Received 30 August 2012; Accepted 1 November 2012

Academic Editor: Nan-Jing Huang

Copyright (C) 2013 S. Plewik and M. Walczyńska. This is an open access article distributed under the Creative Commons Attribution License, which permits unrestricted use, distribution, and reproduction in any medium, provided the original work is properly cited.

\begin{abstract}
The paper fills gaps in knowledge about Kuratowski operations which are already in the literature. The Cayley table for these operations has been drawn up. Techniques, using only paper and pencil, to point out all semigroups and its isomorphism types are applied. Some results apply only to topology, and one cannot bring them out, using only properties of the complement and a closure-like operation. The arguments are by systematic study of possibilities.
\end{abstract}

\section{Introduction}

Let $X$ be a topological space. Denote by $A^{-}$closure of the set $A \subseteq X$. Let $A^{c}$ be the complement of $A$; that is, $X \backslash A=$ $A^{c}$. The aim of this paper is to examine monoids generated under compositions from the closure and the complement. A widely known fact due to Kuratowski [1] states that at most 14 distinct operations can be formed from such compositions. Mark them as follows. Kuratowski operations:

$$
\begin{aligned}
& \sigma_{0}(A)=A \text { (the identity), } \\
& \sigma_{1}(A)=A^{c} \text { (the complement), } \\
& \sigma_{2}(A)=A^{-} \text {(the closure), } \\
& \sigma_{3}(A)=A^{c-}, \\
& \sigma_{4}(A)=A^{-c} \\
& \sigma_{5}(A)=A^{c-c} \text { (the interior), } \\
& \sigma_{6}(A)=A^{-c-}, \\
& \sigma_{7}(A)=A^{c-c-}, \\
& \sigma_{8}(A)=A^{-c-c}, \\
& \sigma_{9}(A)=A^{c-c-c}, \\
& \sigma_{10}(A)=A^{-c-c-}, \\
& \sigma_{11}(A)=A^{c-c-c-} \\
& \sigma_{12}(A)=A^{-c-c-c}, \\
& \sigma_{13}(A)=A^{c-c-c-c} .
\end{aligned}
$$

The following rules was found in the original paper by Kuratowski [1, pages 183-184]. In the Engelking book [2], they are commented by a hint on page 81 . Cancellation rules:

$$
A^{-c-}=A^{-c-c-c-}, \quad A^{c-c-}=A^{c-c-c-c-} .
$$

Kuratowski operations have been studied by several authors, for example, [3] or [4]. A list of some other authors one can find in the paper [5] by Gardner and Jackson. For the first time these operations were systematically studied in the dissertation by Kuratowski, whose results were published in [1]. Tasks relating to these operations are usually resolved at lectures or exercises with general topology. They are normally left to students for independent resolution. For example, determine how many different ways they convert a given set.

This note is organized as follows. Kuratowski operations and their marking are described in the introduction. Their properties of a much broader context than for topologies are presented in Section 2. The Cayley table, for the monoid M of all Kuratowski operations, has been drawn up in Section 3. We hope that this table has not yet been published in the literature. Having this table, one can create a computer program that calculates all the semigroups contained in $\mathbb{M}$. However, in Sections 4-8, we present a framework (i.e., techniques using only paper and pencil) to point out all 118 semigroups and 56 isomorphism types of them. The list of 43 semigroups which are not monoids is presented in Section 9. In this section, also isomorphism types are discussed in order of the number of elements in semigroups. Finally, we present 
cancellation rules (relations) motivated by some topological spaces.

\section{Cancellation Rules}

A map $f: P(X) \rightarrow P(X)$ is called:

(i) increasing, if $A \subseteq B$ implies $f(A) \subseteq f(B)$;

(ii) decreasing, if $A \subseteq B$ implies $f(B) \subseteq f(A)$;

(iii) an involution, if the composition $f \circ f$ is the identity;

(iv) an idempotent, if $f \circ f=f$.

Assume that $A \mapsto \sigma_{0}(A)$ is the identity, $A \mapsto \sigma_{1}(A)$ is a decreasing involution, and $A \mapsto \sigma_{2}(A)$ is an increasing idempotent map. Other operations $\sigma_{i}$ let be compositions of $\sigma_{1}$ and $\sigma_{2}$ as it was with the kuratowski operations. We get the following cancellation rules.

Lemma 1. If $B \subseteq \sigma_{2}(B)$, then

$$
\sigma_{2} \circ \sigma_{12}=\sigma_{6}, \quad \sigma_{2} \circ \sigma_{13}=\sigma_{7} .
$$

Proof. For clarity of this proof, use designations $\sigma_{1}(A)=A^{c}$ and $\sigma_{2}(A)=A^{-}$. Thus, we shall prove

$$
A^{-c-c-c-}=A^{-c-}, \quad A^{c-c-c-c-}=A^{c-c-} .
$$

We start with $A^{-c-c} \subseteq A^{-c-c-}$, substituting $B=A^{-c-c}$ in $B \subseteq$ $B^{-}$. This corresponds to $A^{-c-c-c} \subseteq A^{-c-c c}=A^{-c-}$, since $\sigma_{1}$ is a decreasing involution. Hence $A^{-c-c-c-} \subseteq A^{-c-}$, since $\sigma_{2}$ an increasing idempotent. have

Since $\sigma_{1}$ is decreasing, $\sigma_{2}$ is increasing, and $B \subseteq B^{-}$, we

$$
B^{-c} \subseteq B^{c} \subseteq B^{c-}
$$

Thus $A^{-c-c} \subseteq A^{-c c-}=A^{-}$, if we put $A^{-c}=B$. Again using that $\sigma_{2}$ is increasing and $\sigma_{1}$ is decreasing, we obtain $A^{-c-c-} \subseteq A^{-}$ and then $A^{-c} \subseteq A^{-c-c-c}$. Finally, we get $A^{-c-} \subseteq A^{-c-c-c-}$.

With $A^{c}$ in the place of $A$ in the rule $A^{-c-}=A^{-c-c-c-}$ we get the second rule.

In academic textbooks of general topology, for example, [2, Problem 1.7.1.], one can find a hint suggested to prove the above cancellation rules. Students go like this: steps $A \subseteq A^{-}$ and $A^{c} \subseteq A^{c-}$ lead to $A^{c-c} \subseteq A$; the special case $A^{-c-c-c} \subseteq$ $A^{-c-}$ (of $A^{c-c} \subseteq A$ ) leads to $A^{-c-c-c-} \subseteq A^{-c-}$; steps $A^{-c-c} \subseteq A^{-}$ and $A^{-c-c-} \subseteq A^{-}$lead to $A^{-c-} \subseteq A^{-c-c-c-}$; at the end, use the last step of the proof of Lemma 1 . Note that, the above proofs do not use the axioms of topology as follows:

$$
\begin{aligned}
& \text { (i) } \emptyset=\emptyset^{-} \text {; } \\
& \text { (ii) }(A \cup B)^{-}=A^{-} \cup B^{-} \text {. }
\end{aligned}
$$

In the literature there are articles in which Kuratowski operations are replaced by some other mappings. For example, Koenen [6] considered linear spaces and put $\sigma_{2}(A)$ to be the convex hull of $A$. In fact, Shum [7] considered $\sigma_{2}$ as the closure due to the algebraic operations. Add to this, that these operations can be applied to so-called Fréchet (V) spaces, which were considered in the book [8, pages 3-37].

\section{The Monoid $\mathbb{M}$}

Let $\mathbb{M}$ be the monoid consisting of all Kuratowski operations; that is, there are assumed cancellation rules: $\sigma_{6}=\sigma_{2} \circ \sigma_{12}$ and $\sigma_{7}=\sigma_{2} \circ \sigma_{13}$. Fill in the Cayley table for $\mathbb{M}$, where the row and column marked by the identity are omitted. Similarly as in [9], the factor that labels the row comes first, and that the factor that labels the column is second. For example, $\sigma_{i} \circ \sigma_{k}$ is in the row marked by $\sigma_{i}$ and the column marked by $\sigma_{k}$

\begin{tabular}{c|ccccccccccccc} 
& $\sigma_{1}$ & $\sigma_{2}$ & $\sigma_{3}$ & $\sigma_{4}$ & $\sigma_{5}$ & $\sigma_{6}$ & $\sigma_{7}$ & $\sigma_{8}$ & $\sigma_{9}$ & $\sigma_{10}$ & $\sigma_{11}$ & $\sigma_{12}$ & $\sigma_{13}$ \\
\hline$\sigma_{1}$ & $\sigma_{0}$ & $\sigma_{4}$ & $\sigma_{5}$ & $\sigma_{2}$ & $\sigma_{3}$ & $\sigma_{8}$ & $\sigma_{9}$ & $\sigma_{6}$ & $\sigma_{7}$ & $\sigma_{12}$ & $\sigma_{13}$ & $\sigma_{10}$ & $\sigma_{11}$ \\
$\sigma_{2}$ & $\sigma_{3}$ & $\sigma_{2}$ & $\sigma_{3}$ & $\sigma_{6}$ & $\sigma_{7}$ & $\sigma_{6}$ & $\sigma_{7}$ & $\sigma_{10}$ & $\sigma_{11}$ & $\sigma_{10}$ & $\sigma_{11}$ & $\sigma_{6}$ & $\sigma_{7}$ \\
$\sigma_{3}$ & $\sigma_{2}$ & $\sigma_{6}$ & $\sigma_{7}$ & $\sigma_{2}$ & $\sigma_{3}$ & $\sigma_{10}$ & $\sigma_{11}$ & $\sigma_{6}$ & $\sigma_{7}$ & $\sigma_{6}$ & $\sigma_{7}$ & $\sigma_{10}$ & $\sigma_{11}$ \\
$\sigma_{4}$ & $\sigma_{5}$ & $\sigma_{4}$ & $\sigma_{5}$ & $\sigma_{8}$ & $\sigma_{9}$ & $\sigma_{8}$ & $\sigma_{9}$ & $\sigma_{12}$ & $\sigma_{13}$ & $\sigma_{12}$ & $\sigma_{13}$ & $\sigma_{8}$ & $\sigma_{9}$ \\
$\sigma_{5}$ & $\sigma_{4}$ & $\sigma_{8}$ & $\sigma_{9}$ & $\sigma_{4}$ & $\sigma_{5}$ & $\sigma_{12}$ & $\sigma_{13}$ & $\sigma_{8}$ & $\sigma_{9}$ & $\sigma_{8}$ & $\sigma_{9}$ & $\sigma_{12}$ & $\sigma_{13}$ \\
$\sigma_{6}$ & $\sigma_{7}$ & $\sigma_{6}$ & $\sigma_{7}$ & $\sigma_{10}$ & $\sigma_{11}$ & $\sigma_{10}$ & $\sigma_{11}$ & $\sigma_{6}$ & $\sigma_{7}$ & $\sigma_{6}$ & $\sigma_{7}$ & $\sigma_{10}$ & $\sigma_{11}$ \\
$\sigma_{7}$ & $\sigma_{6}$ & $\sigma_{10}$ & $\sigma_{11}$ & $\sigma_{6}$ & $\sigma_{7}$ & $\sigma_{6}$ & $\sigma_{7}$ & $\sigma_{10}$ & $\sigma_{11}$ & $\sigma_{10}$ & $\sigma_{11}$ & $\sigma_{6}$ & $\sigma_{7}$ \\
$\sigma_{8}$ & $\sigma_{9}$ & $\sigma_{8}$ & $\sigma_{9}$ & $\sigma_{12}$ & $\sigma_{13}$ & $\sigma_{12}$ & $\sigma_{13}$ & $\sigma_{8}$ & $\sigma_{9}$ & $\sigma_{8}$ & $\sigma_{9}$ & $\sigma_{12}$ & $\sigma_{13}$ \\
$\sigma_{9}$ & $\sigma_{8}$ & $\sigma_{12}$ & $\sigma_{13}$ & $\sigma_{8}$ & $\sigma_{9}$ & $\sigma_{8}$ & $\sigma_{9}$ & $\sigma_{12}$ & $\sigma_{13}$ & $\sigma_{12}$ & $\sigma_{13}$ & $\sigma_{8}$ & $\sigma_{9}$ \\
$\sigma_{10}$ & $\sigma_{11}$ & $\sigma_{10}$ & $\sigma_{11}$ & $\sigma_{6}$ & $\sigma_{7}$ & $\sigma_{6}$ & $\sigma_{7}$ & $\sigma_{10}$ & $\sigma_{11}$ & $\sigma_{10}$ & $\sigma_{11}$ & $\sigma_{6}$ & $\sigma_{7}$ \\
$\sigma_{11}$ & $\sigma_{10}$ & $\sigma_{6}$ & $\sigma_{7}$ & $\sigma_{10}$ & $\sigma_{11}$ & $\sigma_{10}$ & $\sigma_{11}$ & $\sigma_{6}$ & $\sigma_{7}$ & $\sigma_{6}$ & $\sigma_{7}$ & $\sigma_{10}$ & $\sigma_{11}$ \\
$\sigma_{12}$ & $\sigma_{13}$ & $\sigma_{12}$ & $\sigma_{13}$ & $\sigma_{8}$ & $\sigma_{9}$ & $\sigma_{8}$ & $\sigma_{9}$ & $\sigma_{12}$ & $\sigma_{13}$ & $\sigma_{12}$ & $\sigma_{13}$ & $\sigma_{8}$ & $\sigma_{9}$ \\
$\sigma_{13}$ & $\sigma_{12}$ & $\sigma_{8}$ & $\sigma_{9}$ & $\sigma_{12}$ & $\sigma_{13}$ & $\sigma_{12}$ & $\sigma_{13}$ & $\sigma_{8}$ & $\sigma_{9}$ & $\sigma_{8}$ & $\sigma_{9}$ & $\sigma_{12}$ & $\sigma_{13}$ \\
& & & & & & & & & & & & &
\end{tabular}

It turns out that the above table allows us to describe all semigroups contained in $\mathbb{M}$, using pencil-and-paper techniques, only. The argument will be by a systematic study of possibilities. Preparing the list of all semigroups consisting of Kuratowski operations we used following principles:

(i) minimal collection of generators is written using $\langle A, B, \ldots, Z\rangle$, where letters denote generators;

(ii) when a semigroup has a few minimal collections of generators, then its name is the first collection in the dictionary order;

(iii) all minimal collections of generators are written with the exception of some containing $\sigma_{0}$;

(iv) we leave to the readers verification that our list is complete, sometimes we add hints.

\section{Semigroups with $\sigma_{1}$}

Observe that each semigroup which contains $\sigma_{0}$ is a monoid. Since $\sigma_{1} \circ \sigma_{1}=\sigma_{0}$, a semigroup which contains $\sigma_{1}$ is a monoid, too.

Theorem 2. There are three monoids containing $\sigma_{1}$ :

(1) $\left\langle\sigma_{1}\right\rangle=\left\{\sigma_{0}, \sigma_{1}\right\}$;

(2) $\mathbb{M}=\left\langle\sigma_{1}, \sigma_{i}\right\rangle=\left\{\sigma_{0}, \sigma_{1}, \ldots, \sigma_{13}\right\}$, where $i \in\{2,3,4,5\}$;

(3) let $\mathbb{M}_{1}=\left\langle\sigma_{1}, \sigma_{6}\right\rangle$. If $j \in\{6,7, \ldots, 13\}$, then

$$
\mathbb{M}_{1}=\left\langle\sigma_{1}, \sigma_{j}\right\rangle=\left\{\sigma_{0}, \sigma_{1}\right\} \cup\left\{\sigma_{6}, \sigma_{7}, \ldots, \sigma_{13}\right\} .
$$

Proof. The equality $\left\langle\sigma_{1}\right\rangle=\left\{\sigma_{0}, \sigma_{1}\right\}$ is obvious.

Since $\sigma_{2}=\sigma_{3} \circ \sigma_{1}=\sigma_{1} \circ \sigma_{4}=\sigma_{1} \circ \sigma_{5} \circ \sigma_{1}$, we have $\left\langle\sigma_{1}, \sigma_{2}\right\rangle=\left\langle\sigma_{1}, \sigma_{3}\right\rangle=\left\langle\sigma_{1}, \sigma_{4}\right\rangle=\left\langle\sigma_{1}, \sigma_{5}\right\rangle=\mathbb{M}$.

If $j \in\{6,7, \ldots, 13\}$, then any composition $\sigma_{j} \circ \sigma_{i}$ or $\sigma_{k} \circ \sigma_{j}$ belongs to $\mathbb{M}_{1}$, and so $\left\langle\sigma_{1}, \sigma_{j}\right\rangle \subseteq \mathbb{M}_{1}$. We have $\sigma_{7}=\sigma_{6} \circ \sigma_{1}$, 
$\sigma_{8}=\sigma_{1} \circ \sigma_{6}, \sigma_{9}=\sigma_{1} \circ \sigma_{7}, \sigma_{10}=\sigma_{6} \circ \sigma_{6}, \sigma_{11}=\sigma_{6} \circ \sigma_{7}$, $\sigma_{12}=\sigma_{8} \circ \sigma_{6}$, and $\sigma_{13}=\sigma_{8} \circ \sigma_{7}$, and so $\mathbb{M}_{1}=\left\langle\sigma_{1}, \sigma_{6}\right\rangle=$ $\left\{\sigma_{0}, \sigma_{1}\right\} \cup\left\{\sigma_{6}, \sigma_{7}, \ldots, \sigma_{13}\right\}$.

Since $\sigma_{6}=\sigma_{7} \circ \sigma_{1}=\sigma_{1} \circ \sigma_{8}=\sigma_{1} \circ \sigma_{9} \circ \sigma_{1}=\sigma_{10} \circ \sigma_{1} \circ \sigma_{10}=$ $\sigma_{11} \circ \sigma_{11} \circ \sigma_{1}=\sigma_{1} \circ \sigma_{12} \circ \sigma_{12}$ and $\sigma_{6}=\sigma_{1} \circ \sigma_{13} \circ \sigma_{1} \circ \sigma_{13} \circ \sigma_{1}$, we have $\left\langle\sigma_{1}, \sigma_{j}\right\rangle=\mathbb{M}_{1}$ for $j \in\{6,7, \ldots, 13\}$.

Consider the permutation

$\left(\begin{array}{llllllllllllll}\sigma_{0} & \sigma_{1} & \sigma_{2} & \sigma_{3} & \sigma_{4} & \sigma_{5} & \sigma_{6} & \sigma_{7} & \sigma_{8} & \sigma_{9} & \sigma_{10} & \sigma_{11} & \sigma_{12} & \sigma_{13} \\ \sigma_{0} & \sigma_{1} & \sigma_{5} & \sigma_{4} & \sigma_{3} & \sigma_{2} & \sigma_{9} & \sigma_{8} & \sigma_{7} & \sigma_{6} & \sigma_{13} & \sigma_{12} & \sigma_{11} & \sigma_{10}\end{array}\right)$.

It determines an automorphism $\mathbb{A}: \mathbb{M} \rightarrow \mathbb{M}$.

Theorem 3. The identity and $\mathbb{A}$ are the only automorphisms of $\mathrm{M}$.

Proof. Delete rows and columns marked by $\sigma_{1}$ in the Cayley table for $\mathbb{M}$. Then, check that the operation $\sigma_{3}$ is in the row or the column marked by $\sigma_{3}$ only. Also, the operation $\sigma_{4}$ is in the row or the column marked by $\sigma_{4}$ only. Therefore the semigroup

$$
\left\langle\sigma_{3}, \sigma_{4}\right\rangle=\left\{\sigma_{2}, \sigma_{3}, \ldots, \sigma_{13}\right\}
$$

has a unique minimal set of generators $\left\{\sigma_{3}, \sigma_{4}\right\}$. The reader is left to check this with the Cayley table for $\mathbb{M}$.

Suppose $\mathbb{G}$ is an automorphism of $\mathbb{M}$. By Theorem 2, $\mathbb{G}$ transforms the set $\left\{\sigma_{2}, \sigma_{3}, \sigma_{4}, \sigma_{5}\right\}$ onto itself. However $\sigma_{2}$ and $\sigma_{5}$ are idempotents, but $\sigma_{3}$ and $\sigma_{4}$ are not idempotents. So, there are two possibilities: $\mathbb{G}\left(\sigma_{3}\right)=\sigma_{3}$ and $\mathbb{G}\left(\sigma_{4}\right)=\sigma_{4}$, which implies that $\mathbb{G}$ is the identity; $\mathbb{G}\left(\sigma_{3}\right)=\sigma_{4}$ and $\mathbb{G}\left(\sigma_{4}\right)=\sigma_{3}$, which implies $\mathbb{G}=\mathbb{A}$. The reader is left to check this with the Cayley table for M. We offer hints: $\sigma_{2}=\sigma_{3} \circ \sigma_{4}, \sigma_{5}=\sigma_{4} \circ \sigma_{3}$, $\sigma_{6}=\sigma_{3} \circ \sigma_{2}, \sigma_{7}=\sigma_{3} \circ \sigma_{3}, \sigma_{8}=\sigma_{4} \circ \sigma_{4}, \sigma_{9}=\sigma_{4} \circ \sigma_{5}$, $\sigma_{10}=\sigma_{6} \circ \sigma_{6}, \sigma_{11}=\sigma_{6} \circ \sigma_{7}, \sigma_{12}=\sigma_{8} \circ \sigma_{6}$, and $\sigma_{13}=\sigma_{8} \circ \sigma_{7}$, to verify the details of this proof.

\section{The Monoid of All Idempotents}

The set $\left\{\sigma_{0}, \sigma_{2}, \sigma_{5}, \sigma_{7}, \sigma_{8}, \sigma_{10}, \sigma_{13}\right\}$ consists of all squares in $\mathbb{M}$. These squares are idempotents and lie on the main diagonal in the Cayley table for M. They constitute a monoid and

$$
\left\{\sigma_{0}, \sigma_{2}, \sigma_{5}, \sigma_{7}, \sigma_{8}, \sigma_{10}, \sigma_{13}\right\}=\left\langle\sigma_{0}, \sigma_{2}, \sigma_{5}\right\rangle .
$$

The permutation

$$
\left(\begin{array}{lllllll}
\sigma_{0} & \sigma_{2} & \sigma_{5} & \sigma_{7} & \sigma_{8} & \sigma_{10} & \sigma_{13} \\
\sigma_{0} & \sigma_{2} & \sigma_{5} & \sigma_{8} & \sigma_{7} & \sigma_{10} & \sigma_{13}
\end{array}\right)
$$

determines the bijection $\square:\left\langle\sigma_{0}, \sigma_{2}, \sigma_{5}\right\rangle \rightarrow\left\langle\sigma_{0}, \sigma_{2}, \sigma_{5}\right\rangle$, such that

$$
\llbracket(\alpha \circ \beta)=\square(\beta) \circ \rrbracket(\alpha),
$$

for any $\alpha, \beta \in\left\langle\sigma_{0}, \sigma_{2}, \sigma_{5}\right\rangle$. To verify this, apply equalities $\sigma_{2} \circ \sigma_{5}=\sigma_{7}, \sigma_{5} \circ \sigma_{2}=\sigma_{8}, \sigma_{2} \circ \sigma_{5} \circ \sigma_{2}=\sigma_{10}$, and $\sigma_{5} \circ \sigma_{2} \circ \sigma_{5}=\sigma_{13}$. Any bijection $\square: G \rightarrow H$, having property $\llbracket(\alpha \circ \beta)=\llbracket(\beta) \triangleright \rrbracket(\alpha)$, transposes Cayley tables for semigroups $G$ and $H$. Several semigroups contained in $\mathbb{M}$ have this property. We leave the reader to verify this.

We shall classify all semigroups contained in the semigroup $\left\langle\sigma_{2}, \sigma_{5}\right\rangle$. Every such semigroup can be extended to a monoid by attaching $\sigma_{0}$ to it. This gives a complete classification of all semigroups in $\left\langle\sigma_{0}, \sigma_{2}, \sigma_{5}\right\rangle$.

The semigroup $\left\langle\sigma_{2}, \sigma_{5}\right\rangle$ contains six groups with exactly one element.

Semigroups $\left\langle\sigma_{2}, \sigma_{10}\right\rangle=\left\{\sigma_{2}, \sigma_{10}\right\}$ and $\left\langle\sigma_{5}, \sigma_{13}\right\rangle=\left\{\sigma_{5}, \sigma_{13}\right\}$ are monoids. Both consist of exactly two elements and are not groups, so they are isomorphic.

Semigroups $\left\langle\sigma_{7}, \sigma_{10}\right\rangle$ and $\left\langle\sigma_{8}, \sigma_{13}\right\rangle$ are isomorphic, in particular $\mathbb{A}\left[\left\langle\sigma_{7}, \sigma_{10}\right\rangle\right]=\left\langle\sigma_{8}, \sigma_{13}\right\rangle$. Also, semigroups $\left\langle\sigma_{7}, \sigma_{13}\right\rangle$ and $\left\langle\sigma_{8}, \sigma_{10}\right\rangle$ are isomorphic by A. Every of these four semigroups has exactly two elements. None of them is a monoid. They form two types of nonisomorphic semigroups, because the bijections $\left\{\left(\sigma_{7}, \sigma_{10}\right),\left(\sigma_{10}, \sigma_{8}\right)\right\}$ and $\left\{\left(\sigma_{7}, \sigma_{8}\right),\left(\sigma_{10}, \sigma_{10}\right)\right\}$ are not isomorphisms.

Semigroups $\left\langle\sigma_{2}, \sigma_{7}\right\rangle=\left\{\sigma_{2}, \sigma_{7}, \sigma_{10}\right\}$ and $\left\langle\sigma_{5}, \sigma_{8}\right\rangle=$ $\left\{\sigma_{5}, \sigma_{8}, \sigma_{13}\right\}$ are isomorphic. Also, semigroups $\left\langle\sigma_{2}, \sigma_{8}\right\rangle=$ $\left\{\sigma_{2}, \sigma_{8}, \sigma_{10}\right\}$ and $\left\langle\sigma_{5}, \sigma_{7}\right\rangle=\left\{\sigma_{5}, \sigma_{7}, \sigma_{13}\right\}$ are isomorphic. In fact, $\mathbb{A}\left[\left\langle\sigma_{2}, \sigma_{7}\right\rangle\right]=\left\langle\sigma_{5}, \sigma_{8}\right\rangle$ and $\mathbb{A}\left[\left\langle\sigma_{2}, \sigma_{8}\right\rangle\right]=\left\langle\sigma_{5}, \sigma_{7}\right\rangle$. None of these semigroups is a monoid. They form two types of nonisomorphic semigroups. Indeed, any isomorphism between $\left\langle\sigma_{2}, \sigma_{7}\right\rangle$ and $\left\langle\sigma_{2}, \sigma_{8}\right\rangle$ must be the identity on the monoid $\left\langle\sigma_{2}, \sigma_{10}\right\rangle$. Therefore would have to be the restriction of $\llbracket$. But $\llbracket$ restricted to $\left\langle\sigma_{7}, \sigma_{10}\right\rangle$ is not an isomorphism.

Semigroups $\left\langle\sigma_{2}, \sigma_{13}\right\rangle=\left\langle\sigma_{2}, \sigma_{7}, \sigma_{8}\right\rangle=\left\{\sigma_{2}, \sigma_{7}, \sigma_{8}, \sigma_{10}, \sigma_{13}\right\}$ and $\left\langle\sigma_{5}, \sigma_{10}\right\rangle=\left\langle\sigma_{5}, \sigma_{7}, \sigma_{8}\right\rangle=\left\{\sigma_{5}, \sigma_{7}, \sigma_{8}, \sigma_{10}, \sigma_{13}\right\}$ are not monoids. They are isomorphic by $\mathbb{A}$.

The semigroup $\left\langle\sigma_{2}, \sigma_{5}\right\rangle$ contains exactly one semigroup with four elements $\left\langle\sigma_{7}, \sigma_{8}\right\rangle=\left\langle\sigma_{10}, \sigma_{13}\right\rangle=\left\{\sigma_{7}, \sigma_{8}, \sigma_{10}, \sigma_{13}\right\}$ which is not a monoid.

Note that $\left\langle\sigma_{2}, \sigma_{5}\right\rangle$ contains twenty different semigroups with nine non-isomorphism types. These are six isomorphic groups with exactly one element $\left\langle\sigma_{2}\right\rangle \cong\left\langle\sigma_{5}\right\rangle \cong\left\langle\sigma_{7}\right\rangle \cong$ $\left\langle\sigma_{8}\right\rangle \cong\left\langle\sigma_{10}\right\rangle \cong\left\langle\sigma_{13}\right\rangle$, two isomorphic monoids with exactly two elements $\left\langle\sigma_{2}, \sigma_{10}\right\rangle \cong\left\langle\sigma_{5}, \sigma_{13}\right\rangle$, two pairs of isomorphic semigroups with exactly two elements $\left\langle\sigma_{7}, \sigma_{10}\right\rangle \cong\left\langle\sigma_{8}, \sigma_{13}\right\rangle$ and $\left\langle\sigma_{7}, \sigma_{13}\right\rangle \cong\left\langle\sigma_{8}, \sigma_{10}\right\rangle$, two pairs of isomorphic semigroups with exactly three elements $\left\langle\sigma_{2}, \sigma_{7}\right\rangle \cong\left\langle\sigma_{5}, \sigma_{8}\right\rangle$ and $\left\langle\sigma_{2}, \sigma_{8}\right\rangle \cong$ $\left\langle\sigma_{5}, \sigma_{7}\right\rangle$, a semigroup with exactly four elements $\left\langle\sigma_{7}, \sigma_{8}\right\rangle$, and two isomorphic semigroups with exactly five elements $\left\langle\sigma_{2}, \sigma_{13}\right\rangle \cong\left\langle\sigma_{5}, \sigma_{10}\right\rangle$ and also $\left\langle\sigma_{2}, \sigma_{5}\right\rangle$. Thus, $\left\langle\sigma_{2}, \sigma_{5}\right\rangle$ contains twenty different semigroups with nine isomorphism types. But $\left\langle\sigma_{0}, \sigma_{2}, \sigma_{5}\right\rangle$ contains forty-one different semigroups with seventeen isomorphism types. Indeed, adding $\sigma_{0}$ to semigroups contained in $\left\langle\sigma_{2}, \sigma_{5}\right\rangle$, which are not monoids, we get twenty monoids with eight non-isomorphism types. Adding $\sigma_{0}$ to a group contained in $\left\langle\sigma_{2}, \sigma_{5}\right\rangle$ we get a monoid isomorphic to $\left\langle\sigma_{2}, \sigma_{10}\right\rangle$.

\section{The Semigroup Consisting of $\left\{\sigma_{6}, \sigma_{7}, \ldots, \sigma_{13}\right\}$}

Using the Cayley table for $\mathbb{M}$, check that

$$
\mathbb{A}\left[\left\{\sigma_{6}, \sigma_{7}, \ldots, \sigma_{13}\right\}\right]=\left\{\sigma_{6}, \sigma_{7}, \ldots, \sigma_{13}\right\} .
$$

Similarly, check that the semigroup $\left\langle\sigma_{6}, \sigma_{9}\right\rangle=\left\{\sigma_{6}, \sigma_{7}, \ldots\right.$, $\left.\sigma_{13}\right\}$ can be represented as $\left\langle\sigma_{6}, \sigma_{13}\right\rangle,\left\langle\sigma_{7}, \sigma_{12}\right\rangle,\left\langle\sigma_{8}, \sigma_{11}\right\rangle$, 
$\left\langle\sigma_{9}, \sigma_{10}\right\rangle$, or $\left\langle\sigma_{11}, \sigma_{12}\right\rangle$. Also $\left\langle\sigma_{6}, \sigma_{9}\right\rangle=\left\langle\sigma_{6}, \sigma_{7}, \sigma_{8}\right\rangle=$ $\left\langle\sigma_{7}, \sigma_{8}, \sigma_{9}\right\rangle=\left\langle\sigma_{10}, \sigma_{11}, \sigma_{13}\right\rangle=\left\langle\sigma_{10}, \sigma_{12}, \sigma_{13}\right\rangle$. These representations exhaust all minimal collections of the Kuratowski operations which generate $\left\langle\sigma_{6}, \sigma_{9}\right\rangle$. Other semigroups included in $\left\langle\sigma_{6}, \sigma_{9}\right\rangle$ have one or two minimal collection of generators. One generator has groups $\left\langle\sigma_{6}\right\rangle=\left\{\sigma_{6}, \sigma_{10}\right\}$, $\left\langle\sigma_{9}\right\rangle=\left\{\sigma_{9}, \sigma_{13}\right\},\left\langle\sigma_{11}\right\rangle=\left\{\sigma_{7}, \sigma_{11}\right\}$, and $\left\langle\sigma_{12}\right\rangle=\left\{\sigma_{8}, \sigma_{12}\right\}$. Each of them has exactly two elements, so they are isomorphic. Semigroups $\left\langle\sigma_{7}, \sigma_{10}\right\rangle,\left\langle\sigma_{7}, \sigma_{13}\right\rangle,\left\langle\sigma_{8}, \sigma_{10}\right\rangle,\left\langle\sigma_{8}, \sigma_{13}\right\rangle$, and $\left\langle\sigma_{7}, \sigma_{8}\right\rangle$ are discussed in the previous section. Contained in $\left\langle\sigma_{6}, \sigma_{9}\right\rangle$ and not previously discussed semigroups are $\left\langle\sigma_{6}, \sigma_{7}\right\rangle$, $\left\langle\sigma_{6}, \sigma_{8}\right\rangle,\left\langle\sigma_{7}, \sigma_{9}\right\rangle$, and $\left\langle\sigma_{8}, \sigma_{9}\right\rangle$. We leave the reader to verify that the following are all possible pairs of Kuratowski operations which constitute a minimal collection of generators for semigroups contained in $\left\langle\sigma_{6}, \sigma_{9}\right\rangle$, but different from the whole. One has

(i) $\left\langle\sigma_{6}, \sigma_{7}\right\rangle=\left\langle\sigma_{6}, \sigma_{11}\right\rangle=\left\langle\sigma_{10}, \sigma_{11}\right\rangle=\left\{\sigma_{6}, \sigma_{7}, \sigma_{10}, \sigma_{11}\right\}$;

(ii) $\left\langle\sigma_{6}, \sigma_{8}\right\rangle=\left\langle\sigma_{6}, \sigma_{12}\right\rangle=\left\langle\sigma_{10}, \sigma_{12}\right\rangle=\left\{\sigma_{6}, \sigma_{8}, \sigma_{10}, \sigma_{12}\right\}$;

(iii) $\left\langle\sigma_{7}, \sigma_{9}\right\rangle=\left\langle\sigma_{9}, \sigma_{11}\right\rangle=\left\langle\sigma_{11}, \sigma_{13}\right\rangle=\left\{\sigma_{7}, \sigma_{9}, \sigma_{11}, \sigma_{13}\right\}$;

(iv) $\left\langle\sigma_{8}, \sigma_{9}\right\rangle=\left\langle\sigma_{9}, \sigma_{12}\right\rangle=\left\langle\sigma_{12}, \sigma_{13}\right\rangle=\left\{\sigma_{8}, \sigma_{9}, \sigma_{12}, \sigma_{13}\right\}$.

Proposition 4. Semigroups $\left\langle\sigma_{6}, \sigma_{7}\right\rangle$ and $\left\langle\sigma_{8}, \sigma_{9}\right\rangle$ are isomorphic, and also semigroups $\left\langle\sigma_{6}, \sigma_{8}\right\rangle$ and $\left\langle\sigma_{7}, \sigma_{9}\right\rangle$ are isomorphic, but semigroups $\left\langle\sigma_{6}, \sigma_{7}\right\rangle,\left\langle\sigma_{6}, \sigma_{8}\right\rangle$ are not isomorphic.

Proof. Isomorphisms are defined by $\mathbb{A}$. Suppose $J:\left\langle\sigma_{6}\right.$, $\left.\sigma_{7}\right\rangle \rightarrow\left\langle\sigma_{6}, \sigma_{8}\right\rangle$ is an isomorphism. Thus $J\left[\left\langle\sigma_{7}, \sigma_{10}\right\rangle\right]=$ $\left\langle\sigma_{8}, \sigma_{10}\right\rangle$. Given $J\left(\sigma_{7}\right)=\sigma_{8}$, we get $\sigma_{10}=J\left(\sigma_{10}\right)=J\left(\sigma_{7}\right.$ 。 $\left.\sigma_{10}\right) \neq \sigma_{8} \circ \sigma_{10}=\sigma_{8}$. But $J\left(\sigma_{7}\right)=\sigma_{10}$ implies $\sigma_{10}=J\left(\sigma_{7}\right)=$ $J\left(\sigma_{10} \circ \sigma_{7}\right) \neq \sigma_{8} \circ \sigma_{10}=\sigma_{8}$. Both possibilities lead to a contradiction.

So, $\left\langle\sigma_{6}, \sigma_{9}\right\rangle$ contains eighteen different semigroups with eight non-isomorphism types. Indeed, these are four isomorphic groups with exactly one element $\left\langle\sigma_{7}\right\rangle \cong\left\langle\sigma_{8}\right\rangle \cong$ $\left\langle\sigma_{10}\right\rangle \cong\left\langle\sigma_{13}\right\rangle$, four isomorphic groups with exactly two elements $\left\langle\sigma_{6}\right\rangle \cong\left\langle\sigma_{9}\right\rangle \cong\left\langle\sigma_{11}\right\rangle \cong\left\langle\sigma_{12}\right\rangle$, two pairs of isomorphic semigroups with exactly two elements $\left\langle\sigma_{7}, \sigma_{10}\right\rangle \cong$ $\left\langle\sigma_{8}, \sigma_{13}\right\rangle$ and $\left\langle\sigma_{7}, \sigma_{13}\right\rangle \cong\left\langle\sigma_{8}, \sigma_{10}\right\rangle$, and five semigroups with exactly four elements $\left\langle\sigma_{6}, \sigma_{7}\right\rangle \cong\left\langle\sigma_{8}, \sigma_{9}\right\rangle,\left\langle\sigma_{6}, \sigma_{8}\right\rangle \cong$ $\left\langle\sigma_{7}, \sigma_{9}\right\rangle,\left\langle\sigma_{7}, \sigma_{8}\right\rangle$, and also $\left\langle\sigma_{6}, \sigma_{9}\right\rangle$.

\section{Remaining Semigroups in $\left\langle\sigma_{3}, \sigma_{4}\right\rangle$}

We have yet to discuss semigroups included in $\left\langle\sigma_{3}, \sigma_{4}\right\rangle$, not included in $\left\langle\sigma_{2}, \sigma_{5}\right\rangle$ and containing at least one of Kuratowski operation $\sigma_{2}, \sigma_{3}, \sigma_{4}$, or $\sigma_{5}$. It will be discussed up to the isomorphism $\mathbb{A}$. Obviously, $\left\langle\sigma_{2}\right\rangle=\left\{\sigma_{2}\right\}$ and $\left\langle\sigma_{5}\right\rangle=\left\{\sigma_{5}\right\}$ are groups.

7.1. Extensions of $\left\langle\boldsymbol{\sigma}_{2}\right\rangle$ and $\left\langle\boldsymbol{\sigma}_{5}\right\rangle$ with Elements of $\left\langle\boldsymbol{\sigma}_{\mathbf{6}}, \boldsymbol{\sigma}_{\mathbf{9}}\right\rangle$. Monoids $\left\langle\sigma_{2}, \sigma_{6}\right\rangle=\left\{\sigma_{2}, \sigma_{6}, \sigma_{10}\right\}$ and $\left\langle\sigma_{2}, \sigma_{10}\right\rangle=\left\{\sigma_{2}, \sigma_{10}\right\}$ have different numbers of elements. Also, $\left\langle\sigma_{2}, \sigma_{6}\right\rangle$ is isomorphic to $\left\langle\sigma_{0}, \sigma_{6}\right\rangle$. Nonisomorphic semigroups $\left\langle\sigma_{2}, \sigma_{7}\right\rangle$ and $\left\langle\sigma_{2}, \sigma_{8}\right\rangle$ are discussed above. The following three semigroups:

(i) $\left\langle\sigma_{2}, \sigma_{11}\right\rangle=\left\langle\sigma_{2}, \sigma_{6}, \sigma_{7}\right\rangle=\left\{\sigma_{2}, \sigma_{6}, \sigma_{7}, \sigma_{10}, \sigma_{11}\right\}$, (ii) $\left\langle\sigma_{2}, \sigma_{12}\right\rangle=\left\langle\sigma_{2}, \sigma_{6}, \sigma_{8}\right\rangle=\left\{\sigma_{2}, \sigma_{6}, \sigma_{8}, \sigma_{10}, \sigma_{12}\right\}$,

(iii) $\left\langle\sigma_{2}, \sigma_{13}\right\rangle=\left\langle\sigma_{2}, \sigma_{7}, \sigma_{8}\right\rangle=\left\{\sigma_{2}, \sigma_{7}, \sigma_{8}, \sigma_{10}, \sigma_{13}\right\}$

are not monoids. They are not isomorphic. Indeed, any isomorphism between these semigroups would lead an isomorphism between $\left\langle\sigma_{6}, \sigma_{7}\right\rangle,\left\langle\sigma_{6}, \sigma_{8}\right\rangle$, or $\left\langle\sigma_{7}, \sigma_{8}\right\rangle$. This is impossible, by Proposition 4 and because $\left\langle\sigma_{7}, \sigma_{8}\right\rangle$ consists of idempotents, but $\sigma_{6}$ is not an idempotent. The nine-element semigroup on the set $\left\{\sigma_{2}, \sigma_{6}, \sigma_{7}, \ldots, \sigma_{13}\right\}$ is represented as $\left\langle\sigma_{2}, \sigma_{9}\right\rangle$. So, we have added four new semigroups, which are not isomorphic with the semigroups previously discussed. These are $\left\langle\sigma_{2}, \sigma_{11}\right\rangle,\left\langle\sigma_{2}, \sigma_{12}\right\rangle,\left\langle\sigma_{2}, \sigma_{13}\right\rangle$, and $\left\langle\sigma_{2}, \sigma_{9}\right\rangle$.

Using $\mathbb{A}$, we have described eight semigroups-each one isomorphic to a semigroup previously discussed-which contains $\sigma_{5}$ and elements (at least one) of $\left\langle\sigma_{6}, \sigma_{9}\right\rangle$. Collections of generators: $\left\langle\sigma_{2}, \sigma_{6}, \sigma_{13}\right\rangle,\left\langle\sigma_{2}, \sigma_{7}, \sigma_{12}\right\rangle,\left\langle\sigma_{2}, \sigma_{8}, \sigma_{11}\right\rangle$, $\left\langle\sigma_{2}, \sigma_{11}, \sigma_{12}\right\rangle,\left\langle\sigma_{2}, \sigma_{11}, \sigma_{13}\right\rangle$, and $\left\langle\sigma_{2}, \sigma_{12}, \sigma_{13}\right\rangle$ are minimal in $\left\langle\sigma_{2}, \sigma_{9}\right\rangle$. Also, collections of generators: $\left\langle\sigma_{5}, \sigma_{7}, \sigma_{12}\right\rangle$, $\left\langle\sigma_{5}, \sigma_{8}, \sigma_{11}\right\rangle,\left\langle\sigma_{5}, \sigma_{9}, \sigma_{10}\right\rangle,\left\langle\sigma_{5}, \sigma_{10}, \sigma_{11}\right\rangle,\left\langle\sigma_{5}, \sigma_{10}, \sigma_{12}\right\rangle$, and $\left\langle\sigma_{5}, \sigma_{11}, \sigma_{12}\right\rangle$ are minimal in $\left\langle\sigma_{5}, \sigma_{6}\right\rangle$.

7.2. Extensions of $\left\langle\boldsymbol{\sigma}_{3}\right\rangle$ and $\left\langle\boldsymbol{\sigma}_{4}\right\rangle$ by Elements from the Semigroup $\left\langle\boldsymbol{\sigma}_{\mathbf{6}}, \boldsymbol{\sigma}_{\mathbf{9}}\right\rangle$. Semigroups $\left\langle\sigma_{3}\right\rangle=\left\{\sigma_{3}, \sigma_{7}, \sigma_{11}\right\}$ and $\left\langle\sigma_{4}\right\rangle=$ $\left\{\sigma_{4}, \sigma_{8}, \sigma_{12}\right\}$ are isomorphic by $\mathbb{A}$. They are not monoids. The semigroup $\left\langle\sigma_{3}\right\rangle$ can be extended using elements of $\left\langle\sigma_{6}, \sigma_{9}\right\rangle$, in three following ways:
(i) $\left\langle\sigma_{3}, \sigma_{6}\right\rangle=\left\langle\sigma_{3}, \sigma_{10}\right\rangle=\left\{\sigma_{3}, \sigma_{6}, \sigma_{7}, \sigma_{10}, \sigma_{11}\right\}$;
(ii) $\left\langle\sigma_{3}, \sigma_{8}\right\rangle=\left\langle\sigma_{3}, \sigma_{12}\right\rangle=\left\{\sigma_{3}, \sigma_{6}, \sigma_{7}, \ldots, \sigma_{13}\right\}$;
(iii) $\left\langle\sigma_{3}, \sigma_{9}\right\rangle=\left\langle\sigma_{3}, \sigma_{13}\right\rangle=\left\{\sigma_{3}, \sigma_{7}, \sigma_{9}, \sigma_{11}, \sigma_{13}\right\}$.

Semigroups $\left\langle\sigma_{3}, \sigma_{6}\right\rangle$ and $\left\langle\sigma_{3}, \sigma_{9}\right\rangle$ are not isomorphic. Indeed, suppose $J:\left\langle\sigma_{3}, \sigma_{6}\right\rangle \rightarrow\left\langle\sigma_{3}, \sigma_{9}\right\rangle$ is an isomorphism. Thus, $J$ is the identity on $\left\langle\sigma_{3}\right\rangle, J\left(\sigma_{6}\right)=\sigma_{9}$, and $J\left(\sigma_{10}\right)=\sigma_{13}$. This gives a contradiction, since $\sigma_{3} \circ \sigma_{6}=\sigma_{10}$ and $\sigma_{3} \circ \sigma_{9}=\sigma_{7}$.

Neither $\left\langle\sigma_{3}, \sigma_{6}\right\rangle$ nor $\left\langle\sigma_{3}, \sigma_{9}\right\rangle$ has a minimal collection of generators with three elements, so they give new isomorphism types. Also, $\left\langle\sigma_{2}, \sigma_{9}\right\rangle$ is not isomorphic to $\left\langle\sigma_{3}, \sigma_{8}\right\rangle$, since $\left\langle\sigma_{2}, \sigma_{9}\right\rangle$ has a unique pair of generators and $\left\langle\sigma_{3}, \sigma_{8}\right\rangle=$ $\left\langle\sigma_{3}, \sigma_{12}\right\rangle$.

Using $\mathbb{A}$, we get-isomorphic to previously discussed ones-semigroups $\left\langle\sigma_{4}, \sigma_{9}\right\rangle=\left\langle\sigma_{4}, \sigma_{13}\right\rangle=\left\{\sigma_{4}, \sigma_{8}, \sigma_{9} \sigma_{12}, \sigma_{13}\right\}$, $\left\langle\sigma_{4}, \sigma_{6}\right\rangle=\left\langle\sigma_{4}, \sigma_{10}\right\rangle=\left\{\sigma_{4}, \sigma_{6}, \sigma_{8} \sigma_{10}, \sigma_{12}\right\}$, and $\left\langle\sigma_{4}, \sigma_{7}\right\rangle=$ $\left\langle\sigma_{4}, \sigma_{11}\right\rangle=\left\{\sigma_{4}, \sigma_{6}, \sigma_{7}, \ldots, \sigma_{13}\right\}$. There exist minimal collections of generators, such as follows:

$$
\begin{aligned}
\left\langle\sigma_{3}, \sigma_{8}\right\rangle & =\left\langle\sigma_{3}, \sigma_{6}, \sigma_{9}\right\rangle=\left\langle\sigma_{3}, \sigma_{6}, \sigma_{13}\right\rangle \\
& =\left\langle\sigma_{3}, \sigma_{9}, \sigma_{10}\right\rangle=\left\langle\sigma_{3}, \sigma_{10}, \sigma_{13}\right\rangle, \\
\left\langle\sigma_{4}, \sigma_{7}\right\rangle & =\left\langle\sigma_{4}, \sigma_{6}, \sigma_{9}\right\rangle=\left\langle\sigma_{4}, \sigma_{9}, \sigma_{10}\right\rangle \\
& =\left\langle\sigma_{4}, \sigma_{6}, \sigma_{13}\right\rangle=\left\langle\sigma_{4}, \sigma_{10}, \sigma_{13}\right\rangle .
\end{aligned}
$$

7.3. More Generators from the Set $\left\{\boldsymbol{\sigma}_{2}, \boldsymbol{\sigma}_{3}, \boldsymbol{\sigma}_{4}, \boldsymbol{\sigma}_{5}\right\}$. Now we check that $\left\langle\sigma_{2}, \sigma_{3}\right\rangle=\left\{\sigma_{2}, \sigma_{3}, \sigma_{6}, \sigma_{7}, \sigma_{10} \sigma_{11}\right\}$ and $\left\langle\sigma_{4}, \sigma_{5}\right\rangle=\left\{\sigma_{4}, \sigma_{5}, \sigma_{8}, \sigma_{9}, \sigma_{12} \sigma_{13}\right\}=\mathbb{A}\left[\left\langle\sigma_{2}, \sigma_{3}\right\rangle\right]$ and also $\left\langle\sigma_{2}, \sigma_{4}\right\rangle=\left\{\sigma_{2}, \sigma_{4}, \sigma_{6}, \sigma_{8}, \sigma_{10} \sigma_{12}\right\}$ and $\left\langle\sigma_{3}, \sigma_{5}\right\rangle=$ $\left\{\sigma_{3}, \sigma_{5}, \sigma_{7}, \sigma_{9}, \sigma_{11} \sigma_{13}\right\}=\mathbb{A}\left[\left\langle\sigma_{2}, \sigma_{4}\right\rangle\right]$ are two pairs of isomorphic semigroups which give two new isomorphism types. 
Each of these semigroups has six element, so in $\mathbb{M}$ there are five six-element semigroups of three isomorphism types, since $\left\langle\sigma_{2}, \sigma_{5}\right\rangle$ has 6 elements which are idempotents.

In $\left\langle\sigma_{3}, \sigma_{4}\right\rangle$ there are seven semigroups which have three generators and have not two generators. These are

(1) $\left\langle\sigma_{2}, \sigma_{3}, \sigma_{8}\right\rangle=\left\langle\sigma_{2}, \sigma_{3}, \sigma_{9}\right\rangle=\left\langle\sigma_{2}, \sigma_{3}, \sigma_{12}\right\rangle=\left\langle\sigma_{2}, \sigma_{3}\right.$, $\left.\sigma_{13}\right\rangle$;

(2) $\left\langle\sigma_{4}, \sigma_{5}, \sigma_{6}\right\rangle=\left\langle\sigma_{4}, \sigma_{5}, \sigma_{7}\right\rangle=\left\langle\sigma_{4}, \sigma_{5}, \sigma_{10}\right\rangle=\left\langle\sigma_{4}, \sigma_{5}\right.$, $\left.\sigma_{11}\right\rangle$;

(3) $\left\langle\sigma_{2}, \sigma_{4}, \sigma_{7}\right\rangle=\left\langle\sigma_{2}, \sigma_{4}, \sigma_{9}\right\rangle=\left\langle\sigma_{2}, \sigma_{4}, \sigma_{11}\right\rangle=\left\langle\sigma_{2}, \sigma_{4}\right.$, $\left.\sigma_{13}\right\rangle$;

(4) $\left\langle\sigma_{3}, \sigma_{5}, \sigma_{6}\right\rangle=\left\langle\sigma_{3}, \sigma_{5}, \sigma_{8}\right\rangle=\left\langle\sigma_{3}, \sigma_{5}, \sigma_{10}\right\rangle=\left\langle\sigma_{3}, \sigma_{5}\right.$, $\left.\sigma_{12}\right\rangle$;

(5) $\left\langle\sigma_{2}, \sigma_{5}, \sigma_{6}\right\rangle=\left\langle\sigma_{2}, \sigma_{5}, \sigma_{9}\right\rangle=\left\langle\sigma_{2}, \sigma_{5}, \sigma_{11}\right\rangle=\left\langle\sigma_{2}, \sigma_{5}\right.$, $\left.\sigma_{12}\right\rangle$

(6) $\left\langle\sigma_{2}, \sigma_{3}, \sigma_{5}\right\rangle$;

(7) $\left\langle\sigma_{2}, \sigma_{4}, \sigma_{5}\right\rangle$.

\section{Semigroups which Are Contained in $\left\langle\sigma_{3}, \sigma_{4}\right\rangle$}

Groups $\left\langle\sigma_{2}\right\rangle \cong\left\langle\sigma_{5}\right\rangle \cong\left\langle\sigma_{7}\right\rangle \cong\left\langle\sigma_{8}\right\rangle \cong\left\langle\sigma_{10}\right\rangle \cong\left\langle\sigma_{13}\right\rangle$ have one element and are isomorphic.

Groups $\left\langle\sigma_{6}\right\rangle \cong\left\langle\sigma_{9}\right\rangle \cong\left\langle\sigma_{11}\right\rangle \cong\left\langle\sigma_{12}\right\rangle$, monoids $\left\langle\sigma_{2}, \sigma_{10}\right\rangle \cong$ $\left\langle\sigma_{5}, \sigma_{13}\right\rangle$, and also semigroups $\left\langle\sigma_{7}, \sigma_{10}\right\rangle \cong\left\langle\sigma_{8}, \sigma_{13}\right\rangle$ and $\left\langle\sigma_{7}, \sigma_{13}\right\rangle \cong\left\langle\sigma_{8}, \sigma_{10}\right\rangle$ have two elements and Cayley tables as follows:

\begin{tabular}{c|llllll} 
& $A$ & $B$ & & & $A$ & $B$ \\
\cline { 2 - 5 }$A$ & $B$ & $A$ & & $A$ & $A$ & $B$ \\
$B$ & $A$ & $B$ & & $B$ & $B$ & $A$ \\
& $A$ & $B$ & & & $A$ & $B$ \\
\hline$A$ & $A$ & $B$ & & $A$ & $A$ & $A$ \\
$B$ & $A$ & $B$ & & $B$ & $B$ & $B$
\end{tabular}

Monoids $\left\langle\sigma_{2}, \sigma_{6}\right\rangle \cong\left\langle\sigma_{5}, \sigma_{9}\right\rangle$, and also semigroups $\left\langle\sigma_{2}, \sigma_{7}\right\rangle \cong\left\langle\sigma_{5}, \sigma_{8}\right\rangle,\left\langle\sigma_{2}, \sigma_{8}\right\rangle \cong\left\langle\sigma_{5}, \sigma_{7}\right\rangle$, and $\left\langle\sigma_{3}\right\rangle \cong\left\langle\sigma_{4}\right\rangle$ have three elements and Cayley tables as follows:

\begin{tabular}{c|llllllll} 
& $A$ & $B$ & $C$ & & & $A$ & $B$ & $C$ \\
\hline$A$ & $A$ & $B$ & $C$ & & $A$ & $A$ & $B$ & $C$ \\
$B$ & $B$ & $C$ & $B$ & & $B$ & $C$ & $B$ & $C$ \\
$C$ & $C$ & $B$ & $C$ & & $C$ & $C$ & $B$ & $C$ \\
& $A$ & $B$ & $C$ & & & $A$ & $B$ & $C$ \\
\hline$A$ & $A$ & $C$ & $C$ & & $A$ & $B$ & $C$ & $B$ \\
$B$ & $B$ & $B$ & $B$ & & $B$ & $C$ & $B$ & $C$ \\
$C$ & $C$ & $C$ & $C$ & & $C$ & $B$ & $C$ & $B$
\end{tabular}

Semigroups $\left\langle\sigma_{6}, \sigma_{7}\right\rangle \cong\left\langle\sigma_{8}, \sigma_{9}\right\rangle,\left\langle\sigma_{6}, \sigma_{8}\right\rangle \cong\left\langle\sigma_{7}, \sigma_{9}\right\rangle$, and $\left\langle\sigma_{7}, \sigma_{8}\right\rangle$ have four elements and Cayley tables as follows:

\begin{tabular}{c|llllllllll} 
& $A$ & $B$ & $C$ & $D$ & & & $A$ & $B$ & $C$ & $D$ \\
\hline$A$ & $C$ & $D$ & $A$ & $B$ & & $A$ & $C$ & $A$ & $A$ & $C$ \\
$B$ & $A$ & $B$ & $C$ & $D$ & & $B$ & $D$ & $B$ & $B$ & $D$ \\
$C$ & $A$ & $B$ & $C$ & $D$ & & $C$ & $A$ & $C$ & $C$ & $A$ \\
$D$ & $C$ & $D$ & $A$ & $B$ & & $D$ & $B$ & $D$ & $D$ & $B$
\end{tabular}

\begin{tabular}{l|llll} 
& $A$ & $B$ & $C$ & $D$ \\
\hline$A$ & $A$ & $C$ & $C$ & $A$ \\
$B$ & $D$ & $B$ & $B$ & $D$ \\
$C$ & $A$ & $C$ & $C$ & $A$ \\
$D$ & $D$ & $B$ & $B$ & $D$
\end{tabular}

Semigroups $\left\langle\sigma_{2}, \sigma_{11}\right\rangle \cong\left\langle\sigma_{5}, \sigma_{12}\right\rangle,\left\langle\sigma_{2}, \sigma_{12}\right\rangle \cong\left\langle\sigma_{5}, \sigma_{11}\right\rangle$, $\left\langle\sigma_{2}, \sigma_{13}\right\rangle \cong\left\langle\sigma_{5}, \sigma_{10}\right\rangle,\left\langle\sigma_{3}, \sigma_{6}\right\rangle \cong\left\langle\sigma_{4}, \sigma_{9}\right\rangle$, and $\left\langle\sigma_{3}, \sigma_{9}\right\rangle \cong$ $\left\langle\sigma_{4}, \sigma_{6}\right\rangle$ have five elements. Semigroups $\left\langle\sigma_{2}, \sigma_{3}\right\rangle \cong\left\langle\sigma_{4}, \sigma_{5}\right\rangle$, $\left\langle\sigma_{2}, \sigma_{4}\right\rangle \cong\left\langle\sigma_{3}, \sigma_{5}\right\rangle$, and $\left\langle\sigma_{2}, \sigma_{5}\right\rangle$ have six elements. Construction of Cayley tables for these semigroups, as well as other semigroups, we leave it to the readers. One can prepare such tables removing from the Cayley table for $\mathbb{M}$ some columns and rows. Then change Kuratowski operations onto letters of the alphabet. For example, $\left\langle\sigma_{0}, \sigma_{2}, \sigma_{5}\right\rangle$ has the following Cayley table:

\begin{tabular}{c|lllllll} 
& 0 & $A$ & $B$ & $C$ & $D$ & $E$ & $F$ \\
\hline 0 & 0 & $A$ & $B$ & $C$ & $D$ & $E$ & $F$ \\
$A$ & $A$ & $A$ & $C$ & $C$ & $E$ & $E$ & $C$ \\
$B$ & $B$ & $D$ & $B$ & $F$ & $D$ & $D$ & $F$ \\
$C$ & $C$ & $E$ & $C$ & $C$ & $E$ & $E$ & $C$ \\
$D$ & $D$ & $D$ & $F$ & $F$ & $D$ & $D$ & $F$ \\
$E$ & $E$ & $E$ & $C$ & $C$ & $E$ & $E$ & $C$ \\
$F$ & $F$ & $D$ & $F$ & $F$ & $D$ & $D$ & $F$
\end{tabular}

Preparing the Cayley table for $\left\langle\sigma_{0}, \sigma_{2}, \sigma_{5}\right\rangle$ we put $\sigma_{0}=0, \sigma_{2}=$ $A, \sigma_{5}=B, \sigma_{7}=C, \sigma_{8}=D, \sigma_{10}=E$, and $\sigma_{13}=F$. This table immediately shows that semigroups $\left\langle\sigma_{2}, \sigma_{5}\right\rangle$ and $\left\langle\sigma_{0}, \sigma_{2}, \sigma_{5}\right\rangle$ have exactly two automorphisms. These are identities and restrictions of $\mathbb{A}$.

Semigroup $\left\langle\sigma_{6}, \sigma_{9}\right\rangle$ has eight elements. Semigroups $\left\langle\sigma_{2}, \sigma_{9}\right\rangle \cong\left\langle\sigma_{5}, \sigma_{6}\right\rangle$ and $\left\langle\sigma_{3}, \sigma_{8}\right\rangle \cong\left\langle\sigma_{4}, \sigma_{7}\right\rangle$ have nine elements. Semigroups $\left\langle\sigma_{2}, \sigma_{3}, \sigma_{8}\right\rangle \cong\left\langle\sigma_{4}, \sigma_{5}, \sigma_{7}\right\rangle,\left\langle\sigma_{2}, \sigma_{4}, \sigma_{7}\right\rangle \cong$ $\left\langle\sigma_{3}, \sigma_{5}, \sigma_{8}\right\rangle$, and $\left\langle\sigma_{2}, \sigma_{5}, \sigma_{6}\right\rangle$ have ten elements. Semigroups $\left\langle\sigma_{2}, \sigma_{3}, \sigma_{5}\right\rangle \cong\left\langle\sigma_{2}, \sigma_{4}, \sigma_{5}\right\rangle$ have eleven elements. In the end, the semigroup $\left\langle\sigma_{3}, \sigma_{4}\right\rangle$ has twelve elements.

Thus, the semigroup $\left\langle\sigma_{3}, \sigma_{4}\right\rangle$ includes fifty-seven semigroups, among which there are ten groups, fourteen monoids, and also forty-three semigroups which are not monoids. These semigroups consist of twenty-eight types of nonisomorphic semigroups, two non-isomorphism types of groups, two non-isomorphism types of monoids which are not groups, and twenty four non-isomorphism types of semigroups which are not monoids.

\section{Viewing Semigroups Contained in $\mathbb{M}$}

9.1. Descriptive Data on Semigroups which Are Contained in M. There are one hundred eighteen, that is, $118=2 \cdot 57+$ 
4, semigroups which are contained in $\mathbb{M}$. These are fiftyseven semigroups contained in $\left\langle\sigma_{3}, \sigma_{4}\right\rangle$, fifty-seven monoids formed by adding $\sigma_{0}$ to a semigroup contained in $\left\langle\sigma_{3}, \sigma_{4}\right\rangle$, groups $\left\langle\sigma_{0}\right\rangle,\left\langle\sigma_{1}\right\rangle$, and monoids $\left\langle\sigma_{1}, \sigma_{6}\right\rangle=\mathbb{M}_{1}$ and $\left\langle\sigma_{1}, \sigma_{2}\right\rangle=$ M.

There are fifty-six types of nonisomorphic semigroups in $\mathbb{M}$. These are twenty-eight non-isomorphism types of semigroups contained in $\left\langle\sigma_{3}, \sigma_{4}\right\rangle$, twenty-six types of nonisomorphic monoids formed by adding $\sigma_{0}$ to a semigroup contained in $\left\langle\sigma_{3}, \sigma_{4}\right\rangle$, and also $\mathbb{M}_{1}$ and $\mathbb{M}$. Indeed, adding $\sigma_{0}$ to a semigroup which is not a monoid we obtain a monoid. In this way, we get twenty-four types of nonisomorphic monoids. Adding $\sigma_{0}$ to a monoid contained in $\left\langle\sigma_{3}, \sigma_{4}\right\rangle$ we get two new non-isomorphism types of monoids. But, adding $\sigma_{0}$ to a group contained in $\left\langle\sigma_{3}, \sigma_{4}\right\rangle$ we get no new type of monoid, since we get a monoid isomorphic to $\left\langle\sigma_{2}, \sigma_{10}\right\rangle$ or $\left\langle\sigma_{2}, \sigma_{6}\right\rangle$. The other two types are $\mathbb{M}_{1}$ and $\mathbb{M}$.

9.2. Semigroups which Are Not Monoids. Below we have reproduced, using the smallest number of generators and the dictionary order, a list of all 43 semigroups, which are included in the $\mathbb{M}$ as follows:

(1) $\left\langle\sigma_{2}, \sigma_{3}\right\rangle=\left\{\sigma_{2}, \sigma_{3}, \sigma_{6}, \sigma_{7}, \sigma_{10}, \sigma_{11}\right\}$.

(2) $\left\langle\sigma_{2}, \sigma_{3}, \sigma_{5}\right\rangle=\left\{\sigma_{2}, \sigma_{3}, \sigma_{5}, \sigma_{6}, \ldots, \sigma_{13}\right\}$.

(3) $\left\langle\sigma_{2}, \sigma_{3}, \sigma_{8}\right\rangle=\left\langle\sigma_{2}, \sigma_{3}, \sigma_{9}\right\rangle=\left\langle\sigma_{2}, \sigma_{3}, \sigma_{12}\right\rangle=\left\langle\sigma_{2}, \sigma_{3}\right.$, $\left.\sigma_{13}\right\rangle=\left\{\sigma_{2}, \sigma_{3}, \sigma_{6}, \sigma_{7}, \ldots, \sigma_{13}\right\}$.

(4) $\left\langle\sigma_{2}, \sigma_{4}\right\rangle=\left\{\sigma_{2}, \sigma_{4}, \sigma_{6}, \sigma_{8}, \sigma_{10}, \sigma_{12}\right\}$.

(5) $\left\langle\sigma_{2}, \sigma_{4}, \sigma_{5}\right\rangle=\left\{\sigma_{2}, \sigma_{4}, \sigma_{5}, \ldots, \sigma_{13}\right\}$.

(6) $\left\langle\sigma_{2}, \sigma_{4}, \sigma_{7}\right\rangle=\left\langle\sigma_{2}, \sigma_{4}, \sigma_{9}\right\rangle=\left\langle\sigma_{2}, \sigma_{4}, \sigma_{11}\right\rangle=\left\langle\sigma_{2}, \sigma_{4}\right.$, $\left.\sigma_{13}\right\rangle=\left\{\sigma_{2}, \sigma_{4}, \sigma_{6}, \sigma_{7} \ldots, \sigma_{13}\right\}$

(7) $\left\langle\sigma_{2}, \sigma_{5}\right\rangle=\left\{\sigma_{2}, \sigma_{5}, \sigma_{7}, \sigma_{8}, \sigma_{10}, \sigma_{13}\right\}$.

(8) $\left\langle\sigma_{2}, \sigma_{5}, \sigma_{6}\right\rangle=\left\langle\sigma_{2}, \sigma_{5}, \sigma_{9}\right\rangle=\left\langle\sigma_{2}, \sigma_{5}, \sigma_{11}\right\rangle=\left\langle\sigma_{2}, \sigma_{5}\right.$, $\left.\sigma_{12}\right\rangle=\left\{\sigma_{2}, \sigma_{5}, \sigma_{6}, \ldots, \sigma_{13}\right\}$.

(9) $\left\langle\sigma_{2}, \sigma_{7}\right\rangle=\left\{\sigma_{2}, \sigma_{7}, \sigma_{10}\right\}$.

(10) $\left\langle\sigma_{2}, \sigma_{8}\right\rangle=\left\{\sigma_{2}, \sigma_{8}, \sigma_{10}\right\}$.

(11) $\left\langle\sigma_{2}, \sigma_{9}\right\rangle=\left\langle\sigma_{2}, \sigma_{6}, \sigma_{13}\right\rangle=\left\langle\sigma_{2}, \sigma_{7}, \sigma_{12}\right\rangle=\left\langle\sigma_{2}, \sigma_{8}\right.$, $\left.\sigma_{11}\right\rangle=\left\langle\sigma_{2}, \sigma_{11}, \sigma_{12}\right\rangle=\left\langle\sigma_{2}, \sigma_{11}, \sigma_{13}\right\rangle=\left\langle\sigma_{2}, \sigma_{12}\right.$, $\left.\sigma_{13}\right\rangle=\left\{\sigma_{2}, \sigma_{6}, \sigma_{7}, \ldots, \sigma_{13}\right\}$.

(12) $\left\langle\sigma_{2}, \sigma_{11}\right\rangle=\left\langle\sigma_{2}, \sigma_{6}, \sigma_{7}\right\rangle=\left\{\sigma_{2}, \sigma_{6}, \sigma_{7}, \sigma_{10}, \sigma_{11}\right\}$.

(13) $\left\langle\sigma_{2}, \sigma_{12}\right\rangle=\left\langle\sigma_{2}, \sigma_{6}, \sigma_{8}\right\rangle=\left\{\sigma_{2}, \sigma_{6}, \sigma_{8}, \sigma_{10}, \sigma_{12}\right\}$.

(14) $\left\langle\sigma_{2}, \sigma_{13}\right\rangle=\left\langle\sigma_{2}, \sigma_{7}, \sigma_{8}\right\rangle=\left\{\sigma_{2}, \sigma_{7}, \sigma_{8}, \sigma_{10}, \sigma_{13}\right\}$.

(15) $\left\langle\sigma_{3}\right\rangle=\left\{\sigma_{3}, \sigma_{7}, \sigma_{11}\right\}$.

(16) $\left\langle\sigma_{3}, \sigma_{4}\right\rangle=\left\{\sigma_{2}, \sigma_{3}, \ldots, \sigma_{13}\right\}$.

(17) $\left\langle\sigma_{3}, \sigma_{5}\right\rangle=\left\{\sigma_{3}, \sigma_{5}, \sigma_{7}, \sigma_{9}, \sigma_{11}, \sigma_{13}\right\}$.

(18) $\left\langle\sigma_{3}, \sigma_{5}, \sigma_{6}\right\rangle=\left\langle\sigma_{3}, \sigma_{5}, \sigma_{8}\right\rangle=\left\langle\sigma_{3}, \sigma_{5}, \sigma_{10}\right\rangle=\left\langle\sigma_{3}, \sigma_{5}\right.$, $\left.\sigma_{12}\right\rangle=\left\{\sigma_{3}, \sigma_{5}, \sigma_{6}, \ldots, \sigma_{13}\right\}$.

(19) $\left\langle\sigma_{3}, \sigma_{6}\right\rangle=\left\langle\sigma_{3}, \sigma_{10}\right\rangle=\left\{\sigma_{3}, \sigma_{6}, \sigma_{7}, \sigma_{10}, \sigma_{11}\right\}$.

(20) $\left\langle\sigma_{3}, \sigma_{8}\right\rangle=\left\langle\sigma_{3}, \sigma_{12}\right\rangle=\left\{\sigma_{3}, \sigma_{6}, \sigma_{7}, \ldots, \sigma_{13}\right\}$.

(21) $\left\langle\sigma_{3}, \sigma_{9}\right\rangle=\left\langle\sigma_{3}, \sigma_{13}\right\rangle=\left\{\sigma_{3}, \sigma_{7}, \sigma_{9}, \sigma_{11}, \sigma_{13}\right\}$.

(22) $\left\langle\sigma_{4}\right\rangle=\left\{\sigma_{4}, \sigma_{8}, \sigma_{12}\right\}$.
(23) $\left\langle\sigma_{4}, \sigma_{5}\right\rangle=\left\{\sigma_{4}, \sigma_{5}, \sigma_{8}, \sigma_{9}, \sigma_{12}, \sigma_{13}\right\}$.

(24) $\left\langle\sigma_{4}, \sigma_{5}, \sigma_{6}\right\rangle=\left\langle\sigma_{4}, \sigma_{5}, \sigma_{7}\right\rangle=\left\langle\sigma_{4}, \sigma_{5}, \sigma_{10}\right\rangle=\left\langle\sigma_{4}, \sigma_{5}\right.$, $\left.\sigma_{11}\right\rangle=\left\{\sigma_{4}, \sigma_{5}, \ldots, \sigma_{13}\right\}$.

(25) $\left\langle\sigma_{4}, \sigma_{6}\right\rangle=\left\langle\sigma_{4}, \sigma_{10}\right\rangle=\left\{\sigma_{4}, \sigma_{6}, \sigma_{8}, \sigma_{10}, \sigma_{12}\right\}$.

(26) $\left\langle\sigma_{4}, \sigma_{7}\right\rangle=\left\langle\sigma_{4}, \sigma_{11}\right\rangle=\left\{\sigma_{4}, \sigma_{6}, \sigma_{7}, \ldots, \sigma_{13}\right\}$.

(27) $\left\langle\sigma_{4}, \sigma_{9}\right\rangle=\left\langle\sigma_{4}, \sigma_{13}\right\rangle=\left\{\sigma_{4}, \sigma_{8}, \sigma_{9}, \sigma_{12}, \sigma_{13}\right\}$.

(28) $\left\langle\sigma_{5}, \sigma_{6}\right\rangle=\left\langle\sigma_{5}, \sigma_{7}, \sigma_{12}\right\rangle=\left\langle\sigma_{5}, \sigma_{8}, \sigma_{11}\right\rangle=\left\langle\sigma_{5}, \sigma_{9}\right.$, $\left.\sigma_{10}\right\rangle=\left\langle\sigma_{5}, \sigma_{10}, \sigma_{11}\right\rangle=\left\langle\sigma_{5}, \sigma_{10}, \sigma_{12}\right\rangle=\left\langle\sigma_{5}, \sigma_{11}\right.$, $\left.\sigma_{12}\right\rangle=\left\{\sigma_{5}, \sigma_{6}, \ldots, \sigma_{13}\right\}$.

(29) $\left\langle\sigma_{5}, \sigma_{7}\right\rangle=\left\{\sigma_{5}, \sigma_{7}, \sigma_{13}\right\}$.

(30) $\left\langle\sigma_{5}, \sigma_{8}\right\rangle=\left\{\sigma_{5}, \sigma_{8}, \sigma_{13}\right\}$.

(31) $\left\langle\sigma_{5}, \sigma_{10}\right\rangle=\left\langle\sigma_{5}, \sigma_{7}, \sigma_{8}\right\rangle=\left\{\sigma_{5}, \sigma_{7}, \sigma_{8}, \sigma_{10}, \sigma_{13}\right\}$.

(32) $\left\langle\sigma_{5}, \sigma_{11}\right\rangle=\left\langle\sigma_{5}, \sigma_{7}, \sigma_{9}\right\rangle=\left\{\sigma_{5}, \sigma_{7}, \sigma_{9}, \sigma_{11}, \sigma_{13}\right\}$.

(33) $\left\langle\sigma_{5}, \sigma_{12}\right\rangle=\left\langle\sigma_{5}, \sigma_{8}, \sigma_{9}\right\rangle=\left\{\sigma_{5}, \sigma_{8}, \sigma_{9}, \sigma_{12}, \sigma_{13}\right\}$.

(34) $\left\langle\sigma_{6}, \sigma_{7}\right\rangle=\left\langle\sigma_{6}, \sigma_{11}\right\rangle=\left\langle\sigma_{10}, \sigma_{11}\right\rangle=\left\{\sigma_{6}, \sigma_{7}, \sigma_{10}, \sigma_{11}\right\}$.

(35) $\left\langle\sigma_{6}, \sigma_{8}\right\rangle=\left\langle\sigma_{6}, \sigma_{12}\right\rangle=\left\langle\sigma_{10}, \sigma_{12}\right\rangle=\left\{\sigma_{6}, \sigma_{8}, \sigma_{10}, \sigma_{12}\right\}$.

(36) $\left\langle\sigma_{6}, \sigma_{9}\right\rangle=\left\langle\sigma_{6}, \sigma_{13}\right\rangle=\left\langle\sigma_{7}, \sigma_{12}\right\rangle=\left\langle\sigma_{8}, \sigma_{11}\right\rangle=\left\langle\sigma_{9}\right.$, $\left.\sigma_{10}\right\rangle=\left\langle\sigma_{11}, \sigma_{12}\right\rangle=\left\langle\sigma_{6}, \sigma_{7}, \sigma_{8}\right\rangle=\left\langle\sigma_{7}, \sigma_{8}, \sigma_{9}\right\rangle=$ $\left\langle\sigma_{10}, \sigma_{11}, \sigma_{13}\right\rangle=\left\langle\sigma_{10}, \sigma_{12}, \sigma_{13}\right\rangle=\left\{\sigma_{6}, \sigma_{7}, \ldots, \sigma_{13}\right\}$.

(37) $\left\langle\sigma_{7}, \sigma_{8}\right\rangle=\left\{\sigma_{7}, \sigma_{8}, \sigma_{10}, \sigma_{13}\right\}$.

(38) $\left\langle\sigma_{7}, \sigma_{9}\right\rangle=\left\{\sigma_{7}, \sigma_{9}, \sigma_{11}, \sigma_{13}\right\}$.

(39) $\left\langle\sigma_{7}, \sigma_{10}\right\rangle=\left\{\sigma_{7}, \sigma_{10}\right\}$.

(40) $\left\langle\sigma_{7}, \sigma_{13}\right\rangle=\left\{\sigma_{7}, \sigma_{13}\right\}$.

(41) $\left\langle\sigma_{8}, \sigma_{9}\right\rangle=\left\{\sigma_{8}, \sigma_{9}, \sigma_{12}, \sigma_{13}\right\}$.

(42) $\left\langle\sigma_{8}, \sigma_{10}\right\rangle=\left\{\sigma_{8}, \sigma_{10}\right\}$.

(43) $\left\langle\sigma_{8}, \sigma_{13}\right\rangle=\left\{\sigma_{8}, \sigma_{13}\right\}$.

9.3. Isomorphism Types of Semigroups Contained in M. Systematize the list of all isomorphism types of semigroups contained in the monoid $\mathbb{M}$. Isomorphisms, which are restrictions of the isomorphism $\mathbb{A}$, will be regarded as self-evident, and therefore they will not be commented on.

(i) The monoid $\mathbb{M}$ contains 12 groups with 2 isomorphism types. These are 7 one-element groups and 5 two-element groups.

(ii) The monoid $\mathbb{M}$ contains 8 two-element monoids with 2 isomorphism types. These are $\sigma_{0}$ added to 6 oneelement groups and $\left\langle\sigma_{2}, \sigma_{10}\right\rangle \cong\left\langle\sigma_{5}, \sigma_{13}\right\rangle$. Also, it contains 4 two-element semigroups, not monoids, with 2 isomorphism types. These are $\left\langle\sigma_{7}, \sigma_{10}\right\rangle \cong$ $\left\langle\sigma_{8}, \sigma_{13}\right\rangle$ and $\left\langle\sigma_{8}, \sigma_{10}\right\rangle \cong\left\langle\sigma_{7}, \sigma_{13}\right\rangle$.

(iii) The monoid $\mathbb{M}$ contains 12 three-element monoids with 5 isomorphism types. These are $\sigma_{0}$ added to 4 two-element groups and also $\left\langle\sigma_{0}, \sigma_{2}, \sigma_{10}\right\rangle \cong$ $\left\langle\sigma_{0}, \sigma_{5}, \sigma_{13}\right\rangle,\left\langle\sigma_{0}, \sigma_{7}, \sigma_{10}\right\rangle \cong\left\langle\sigma_{0}, \sigma_{8}, \sigma_{13}\right\rangle$, $\left\langle\sigma_{0}, \sigma_{8}, \sigma_{10}\right\rangle \cong\left\langle\sigma_{0}, \sigma_{7}, \sigma_{13}\right\rangle$, and $\left\langle\sigma_{2}, \sigma_{6}\right\rangle \cong\left\langle\sigma_{5}, \sigma_{9}\right\rangle$.

(iv) The monoid $\mathbb{M}$ contains 6 three-element semigroups, not monoids, with 3 isomorphism types. These are $\left\langle\sigma_{2}, \sigma_{7}\right\rangle \cong\left\langle\sigma_{5}, \sigma_{8}\right\rangle,\left\langle\sigma_{2}, \sigma_{8}\right\rangle \cong\left\langle\sigma_{5}, \sigma_{7}\right\rangle$, and $\left\langle\sigma_{3}\right\rangle \cong$ $\left\langle\sigma_{4}\right\rangle$. 
(v) The monoid $\mathbb{M}$ contains 8 four-element monoids, each contains $\sigma_{0}$, with 4 isomorphism types. These are semigroups from two preceding items that can be substantially extended by $\sigma_{0}$.

(vi) The monoid $\mathbb{M}$ contains 5 four-element semigroups, not monoids, with 3 isomorphism types. These are $\left\langle\sigma_{6}, \sigma_{7}\right\rangle \cong\left\langle\sigma_{8}, \sigma_{9}\right\rangle,\left\langle\sigma_{6}, \sigma_{8}\right\rangle \cong\left\langle\sigma_{7}, \sigma_{9}\right\rangle$ and $\left\langle\sigma_{7}, \sigma_{8}\right\rangle$. These semigroups extended by $\sigma_{0}$ yield 5 monoids, all which consist of five elements, with 3 isomorphism types.

(vii) The monoid M contains 10 five-element semigroupsnot monoids, with 5 isomorphism types. These are $\left\langle\sigma_{2}, \sigma_{11}\right\rangle \cong\left\langle\sigma_{5}, \sigma_{12}\right\rangle,\left\langle\sigma_{2}, \sigma_{12}\right\rangle \cong\left\langle\sigma_{5}, \sigma_{11}\right\rangle,\left\langle\sigma_{2}, \sigma_{13}\right\rangle \cong$ $\left\langle\sigma_{5}, \sigma_{10}\right\rangle,\left\langle\sigma_{3}, \sigma_{6}\right\rangle \cong\left\langle\sigma_{4}, \sigma_{9}\right\rangle$, and $\left\langle\sigma_{3}, \sigma_{9}\right\rangle \cong\left\langle\sigma_{4}, \sigma_{6}\right\rangle$. These semigroups extended by $\sigma_{0}$ yield 10 monoids, all of which consist of six elements, with 5 isomorphism types. We get 10 new isomorphism types, since the semigroups are distinguished by semigroups $\left\langle\sigma_{3}\right\rangle$ and $\left\langle\sigma_{4}\right\rangle$, and by nonisomorphic semigroups $\left\langle\sigma_{6}, \sigma_{7}\right\rangle$, $\left\langle\sigma_{6}, \sigma_{8}\right\rangle$, and $\left\langle\sigma_{7}, \sigma_{8}\right\rangle$.

(viii) The monoid $\mathbb{M}$ contains 5 six-element semigroups, not monoids, with 3 isomorphism types. These are $\left\langle\sigma_{2}, \sigma_{3}\right\rangle \cong\left\langle\sigma_{4}, \sigma_{5}\right\rangle,\left\langle\sigma_{2}, \sigma_{4}\right\rangle \cong\left\langle\sigma_{3}, \sigma_{5}\right\rangle$, and $\left\langle\sigma_{2}, \sigma_{5}\right\rangle$. These semigroups extended by $\sigma_{0}$ yield 5 monoids, all of which consist of seven elements, with 3 isomorphism types. We get 6 new isomorphism types, since the semigroups are distinguished by non isomorphic semigroups $\left\langle\sigma_{6}, \sigma_{7}\right\rangle,\left\langle\sigma_{6}, \sigma_{8}\right\rangle$, and $\left\langle\sigma_{7}, \sigma_{8}\right\rangle$.

(ix) The monoid $\mathbb{M}$ contains no seven-element semigroup, not a monoid, no eight-element monoid and the only semigroup $\left\langle\sigma_{6}, \sigma_{9}\right\rangle$ with exactly eight elements and the only monoid $\left\langle\sigma_{0}, \sigma_{6}, \sigma_{9}\right\rangle$ with exactly nine elements.

(x) The monoid $\mathbb{M}$ contains 4 nine-element semigroups, not monoids, with 2 isomorphism types. These are $\left\langle\sigma_{2}, \sigma_{9}\right\rangle \cong\left\langle\sigma_{5}, \sigma_{6}\right\rangle$ and $\left\langle\sigma_{3}, \sigma_{8}\right\rangle \cong\left\langle\sigma_{4}, \sigma_{7}\right\rangle$. The semigroup $\left\langle\sigma_{2}, \sigma_{9}\right\rangle$ does not contain a semigroup isomorphic to $\left\langle\sigma_{3}\right\rangle$, hence it is not isomorphic to $\left\langle\sigma_{3}, \sigma_{8}\right\rangle$. These semigroups extended by $\sigma_{0}$ yield 4 monoids, all of which consist of ten elements, with 2 isomorphism types.

(xi) The monoid $\mathbb{M}$ contains 6 ten-element semigroups, not monoids, with 4 isomorphism types. These are $\left\langle\sigma_{1}, \sigma_{6}\right\rangle,\left\langle\sigma_{2}, \sigma_{3}, \sigma_{8}\right\rangle \cong\left\langle\sigma_{4}, \sigma_{5}, \sigma_{6}\right\rangle$, $\left\langle\sigma_{2}, \sigma_{4}, \sigma_{7}\right\rangle \cong\left\langle\sigma_{3}, \sigma_{5}, \sigma_{6}\right\rangle$, and $\left\langle\sigma_{2}, \sigma_{5}, \sigma_{6}\right\rangle$. These semigroups (except $\left\langle\sigma_{1}, \sigma_{6}\right\rangle$ ) extended by $\sigma_{0}$ yield 5 monoids, all of which consist of ten elements, with 3 isomorphism types. We get 6 new isomorphism types, since the semigroups are distinguished by not isomorphic semigroups $\left\langle\sigma_{2}, \sigma_{3}\right\rangle,\left\langle\sigma_{2}, \sigma_{4}\right\rangle$, and $\left\langle\sigma_{2}, \sigma_{5}\right\rangle$.

(xii) The monoid $\mathbb{M}$ contains 2 isomorphic semigroups, not monoids, which consist of 11 elements, that is, $\left\langle\sigma_{2}, \sigma_{3}, \sigma_{5}\right\rangle \cong\left\langle\sigma_{2}, \sigma_{4}, \sigma_{5}\right\rangle$. These semigroups extended by $\sigma_{0}$ yield 2 isomorphic monoids, which consist of 12 elements. The monoid $\mathbb{M}$ contains no larger semigroup with the exception of itself.

\section{Cancellation Rules Motivated by Some Topological Properties}

10.1. Some Consequences of the Axiom $\emptyset=\emptyset^{-}$. So far, we used only the following relations (above named cancellation rules): $\sigma_{2} \circ \sigma_{2}=\sigma_{2}, \sigma_{1} \circ \sigma_{1}=\sigma_{0}, \sigma_{2} \circ \sigma_{12}=\sigma_{6}$, and $\sigma_{2} \circ \sigma_{13}=\sigma_{7}$. When one assumes $X \neq \emptyset=\emptyset^{-}$, then

$$
\begin{aligned}
X & =\sigma_{0}(X)=\sigma_{2}(X)=\sigma_{5}(X)=\sigma_{7}(X) \\
& =\sigma_{8}(X)=\sigma_{10}(X)=\sigma_{13}(X), \\
\emptyset & =\sigma_{1}(X)=\sigma_{3}(X)=\sigma_{4}(X)=\sigma_{6}(X) \\
& =\sigma_{9}(X)=\sigma_{11}(X)=\sigma_{12}(X) .
\end{aligned}
$$

Using the substitution $A \mapsto A^{c}$, one obtains equivalent relations between operations from the set $\left\{\sigma_{1}, \sigma_{3}\right.$, $\left.\sigma_{4}, \sigma_{6}, \sigma_{9}, \sigma_{11}, \sigma_{12}\right\}$, and conversely. Therefore, cancellation rules are topologically reasonable only between the operations from the monoid as follows:

$$
\left\langle\sigma_{0}, \sigma_{2}, \sigma_{5}\right\rangle=\left\{\sigma_{0}, \sigma_{2}, \sigma_{5}, \sigma_{7}, \sigma_{8}, \sigma_{10}, \sigma_{13}\right\} .
$$

Chapman, see [3], considered properties of subsets with respect to such relations. Below, we are going to identify relations that are determined by some topological spaces; compare $[10,11]$.

10.2. The Relation $\sigma_{0}=\sigma_{2}$. If a topological space $X$ is discrete, then there exist two Kuratowski operation, only. These are $\sigma_{0}$ and $\sigma_{1}$. So, the monoid of Kuratowski operations reduced to the group $\left\langle\sigma_{1}\right\rangle$.

The relation $\sigma_{0}=\sigma_{2}$ is equivalent to any relation $\sigma_{0}=$ $\sigma_{i}$, where $i \in\{5,7,8,10,13\}$. Any such relation implies that every subset of $X$ has to be closed and open; that is, $X$ has to be discrete. However, one can check these using (only) the facts that $\sigma_{1}$ is an involution and $\sigma_{2}$ is an idempotent and the cancellation rules, that is, the Cayley table for M. So, $\sigma_{0}=\sigma_{2}$ follows

$$
\sigma_{0}=\sigma_{2}=\sigma_{5}=\sigma_{7}=\sigma_{8}=\sigma_{10}=\sigma_{13} .
$$

10.3. The Relation $\boldsymbol{\sigma}_{2}=\boldsymbol{\sigma}_{5}$. Topologically, $\sigma_{2}=\sigma_{5}$ means that $X$ must be discrete. This is so because $A^{c-c} \subseteq A \subseteq A^{-}$ for any $A \subseteq X$.

10.4. The Relation $\boldsymbol{\sigma}_{2}=\boldsymbol{\sigma}_{7}$. Topologically, the relation $\sigma_{2}=$ $\sigma_{7}$ implies $\sigma_{0}=\sigma_{2}$. But it requires the use of topology axioms $\emptyset=\emptyset^{-}$and $C^{-} \cup B^{-}=(C \cup B)^{-}$for each $C$ and $B$.

Lemma 5. For any topological space $\sigma_{2}=\sigma_{7}$ implies $\sigma_{2}=\sigma_{8}$.

Proof. If $\sigma_{2}=\sigma_{7}$, then $A \neq \emptyset \Rightarrow A^{c-c} \neq \emptyset$, for any $A \subseteq X$. Indeed, if $A^{c-c}=\emptyset$, then $\sigma_{7}(A)=\emptyset^{-}=\emptyset$. Since $A \neq \emptyset$, then $\sigma_{2}(A) \neq \emptyset$. Hence $\sigma_{2}(A) \neq \sigma_{7}(A)$, a contradiction.

The axiom $C^{-} \cup B^{-}=(C \cup B)^{-}$implies that always

$$
\left(A^{-} \cap A^{-c-}\right)^{c-c}=\emptyset .
$$

Thus, the additional assumption $\sigma_{2}=\sigma_{7}$ follows that always $A^{-} \cap A^{-c-}=\emptyset$. Therefore, always $A^{-c-}=A^{-c}$, but this means 
that any closed set has to be open; in other words, $\sigma_{2}=\sigma_{8}$.

Proposition 6. For any topological space $\sigma_{2}=\sigma_{7}$ implies $\sigma_{0}=$ $\sigma_{2}$.

Proof. But the relation $\sigma_{2}=\sigma_{7}$ is equivalent with $\sigma_{5}=\sigma_{8}$. By Lemma 5, we get $\sigma_{2}=\sigma_{5}$. Finally $\sigma_{0}=\sigma_{2}$.

The relation $\sigma_{2}=\sigma_{7}$ has interpretation without the axiom $\emptyset=\emptyset^{-}$. Indeed, suppose $X=\{a, b\}$. Put

$$
\sigma_{2}(\emptyset)=\{a\}=\sigma_{2}(\{a\}), \quad X=\sigma_{2}(X)=\sigma_{2}(\{b\}) .
$$

Then, check that $\sigma_{2}=\sigma_{7}$ and

$$
\sigma_{8}(\emptyset)=\sigma_{5}(\{a\})=\sigma_{4}(\{b\})=\sigma_{1}(X)=\emptyset ;
$$

in other words, $\sigma_{2}=\sigma_{7}$ and $\sigma_{2} \neq \sigma_{8}$. However, $\sigma_{2}=\sigma_{7}$ is equivalent to $\sigma_{5}=\sigma_{8}$.

This relation implies $\sigma_{2}=\sigma_{7}=\sigma_{10}, \sigma_{5}=\sigma_{8}=\sigma_{13}$, $\sigma_{3}=\sigma_{6}=\sigma_{11}$, and $\sigma_{4}=\sigma_{9}=\sigma_{12}$. For this interpretation, the monoid $\mathbb{M} /\left(\sigma_{2}=\sigma_{7}\right)$, consisting of Kuratowski operation over a such $X$, has six elements, only. In $\mathbb{M} /\left(\sigma_{2}=\sigma_{7}\right)$, there are relations covered by the following proposition, only.

Proposition 7. For any monoid with the Cayley table as for $\mathbb{M}$, the relation $\sigma_{2}=\sigma_{7}$ implies

(i) $\sigma_{2}=\sigma_{7}=\sigma_{10}=\sigma_{7} \circ \sigma_{2}$;

(ii) $\sigma_{5}=\sigma_{1} \circ \sigma_{2} \circ \sigma_{1}=\sigma_{1} \circ \sigma_{7} \circ \sigma_{1}=\sigma_{8}=\sigma_{5} \circ \sigma_{2}=\sigma_{5} \circ \sigma_{7}=$ $\sigma_{13}$

(iii) $\sigma_{3}=\sigma_{2} \circ \sigma_{1}=\sigma_{7} \circ \sigma_{1}=\sigma_{6}=\sigma_{10} \circ \sigma_{1}=\sigma_{11}$;

(iv) $\sigma_{4}=\sigma_{1} \circ \sigma_{2}=\sigma_{1} \circ \sigma_{7}=\sigma_{9}=\sigma_{1} \circ \sigma_{10}=\sigma_{12}$.

Thus, the Cayley table does not contain the complete information resulting from the axioms of topology.

10.5. The Relation $\boldsymbol{\sigma}_{\mathbf{2}}=\boldsymbol{\sigma}_{\mathbf{8}}$. Topologically, the relation $\sigma_{2}=$ $\sigma_{8}$ means that any closed set is open, too. Thus, if $X=\{a, b, c\}$ is a topological space with the open sets $X, \emptyset,\{a, b\}$, and $\{c\}$, then $\mathbb{M} /\left(\sigma_{2}=\sigma_{8}\right)$ is the monoid of all Kuratowski operations over $X$. Relations $\sigma_{2}=\sigma_{8}$ and $\sigma_{5}=\sigma_{7}$ are equivalent. They imply relations: $\sigma_{2}=\sigma_{8}=\sigma_{10}, \sigma_{5}=\sigma_{7}=\sigma_{13}, \sigma_{3}=\sigma_{9}=\sigma_{11}$, and $\sigma_{4}=\sigma_{6}=\sigma_{12}$. The permutation

$$
\left(\begin{array}{llllll}
\sigma_{0} & \sigma_{1} & \sigma_{2} & \sigma_{3} & \sigma_{4} & \sigma_{5} \\
\sigma_{0} & \sigma_{1} & \sigma_{5} & \sigma_{4} & \sigma_{3} & \sigma_{2}
\end{array}\right)
$$

determines the isomorphism between monoids $\mathbb{M} /\left(\sigma_{2}=\sigma_{7}\right)$ and $\mathbb{M} /\left(\sigma_{2}=\sigma_{8}\right)$.

10.6. The Relations $\sigma_{2}=\sigma_{10}$ and $\sigma_{2}=\sigma_{13}$. Topologically, the relation $\sigma_{2}=\sigma_{8}$ means that any nonempty closed set has nonempty interior. For each $A$, the closed set $A^{-} \cap A^{-c-}$ has empty interior, so the relation $\sigma_{2}=\sigma_{10}$ implies that $A^{-c}$ is closed. Hence, any open set has to be closed, so it implies $\sigma_{2}=\sigma_{8}$. The relation $\sigma_{2}=\sigma_{13}$ follows that each closed set has to be open, so it implies $\sigma_{2}=\sigma_{8}$, too.
10.7. The Relation $\sigma_{7}=\sigma_{8}$. Using the Cayley table for $\mathbb{M}$, one can check that the relations $\sigma_{7}=\sigma_{8}$ and $\sigma_{10}=\sigma_{13}$ are equivalent. Each of them gives $\sigma_{7}=\sigma_{8}=\sigma_{10}=\sigma_{13}$ and $\sigma_{6}=\sigma_{9}=\sigma_{11}=\sigma_{12}$. If $X=\{a, b\}$ is a topological space with the open sets $X, \emptyset$, and $\{a\}$, then $\mathbb{M} /\left(\sigma_{7}=\sigma_{8}\right)$ is the monoid of all Kuratowski operations over $X$ and consists of 8 elements.

10.8. The Relation $\boldsymbol{\sigma}_{\mathbf{7}}=\boldsymbol{\sigma}_{\mathbf{1 0}}$. Using the Cayley table for $\mathbb{M}$, one can check that the relations $\sigma_{7}=\sigma_{10}, \sigma_{8}=\sigma_{13}, \sigma_{6}=\sigma_{11}$, and $\sigma_{9}=\sigma_{12}$ are equivalent. If $X$ is a sequence converging to the point $g$ and $g \in X$, then $\mathbb{M} /\left(\sigma_{7}=\sigma_{10}\right)$ is the monoid of all Kuratowski operations over $X$ and consists of 10 elements.

10.9. The Relation $\boldsymbol{\sigma}_{7}=\sigma_{13}$. Using the Cayley table for $\mathbb{M}$, one can check that the relations $\sigma_{7}=\sigma_{13}$ and $\sigma_{8}=\sigma_{10}$ are equivalent. Also $\sigma_{6}=\sigma_{12}$ and $\sigma_{9}=\sigma_{11}$. These relations give the monoid with the following Cayley table, where the row and column marked by the identity are omitted:

\begin{tabular}{c|ccccccccc} 
& $\sigma_{1}$ & $\sigma_{2}$ & $\sigma_{3}$ & $\sigma_{4}$ & $\sigma_{5}$ & $\sigma_{6}$ & $\sigma_{7}$ & $\sigma_{8}$ & $\sigma_{9}$ \\
\hline$\sigma_{1}$ & $\sigma_{0}$ & $\sigma_{4}$ & $\sigma_{5}$ & $\sigma_{2}$ & $\sigma_{3}$ & $\sigma_{8}$ & $\sigma_{9}$ & $\sigma_{6}$ & $\sigma_{7}$ \\
$\sigma_{2}$ & $\sigma_{3}$ & $\sigma_{2}$ & $\sigma_{3}$ & $\sigma_{6}$ & $\sigma_{7}$ & $\sigma_{6}$ & $\sigma_{7}$ & $\sigma_{8}$ & $\sigma_{9}$ \\
$\sigma_{3}$ & $\sigma_{2}$ & $\sigma_{6}$ & $\sigma_{7}$ & $\sigma_{2}$ & $\sigma_{3}$ & $\sigma_{8}$ & $\sigma_{9}$ & $\sigma_{6}$ & $\sigma_{7}$ \\
$\sigma_{4}$ & $\sigma_{5}$ & $\sigma_{4}$ & $\sigma_{5}$ & $\sigma_{8}$ & $\sigma_{9}$ & $\sigma_{8}$ & $\sigma_{9}$ & $\sigma_{6}$ & $\sigma_{7}$ \\
$\sigma_{5}$ & $\sigma_{4}$ & $\sigma_{8}$ & $\sigma_{9}$ & $\sigma_{4}$ & $\sigma_{5}$ & $\sigma_{6}$ & $\sigma_{7}$ & $\sigma_{8}$ & $\sigma_{9}$ \\
$\sigma_{6}$ & $\sigma_{7}$ & $\sigma_{6}$ & $\sigma_{7}$ & $\sigma_{8}$ & $\sigma_{9}$ & $\sigma_{8}$ & $\sigma_{9}$ & $\sigma_{6}$ & $\sigma_{7}$ \\
$\sigma_{7}$ & $\sigma_{6}$ & $\sigma_{8}$ & $\sigma_{9}$ & $\sigma_{6}$ & $\sigma_{7}$ & $\sigma_{6}$ & $\sigma_{7}$ & $\sigma_{8}$ & $\sigma_{9}$ \\
$\sigma_{8}$ & $\sigma_{9}$ & $\sigma_{8}$ & $\sigma_{9}$ & $\sigma_{6}$ & $\sigma_{7}$ & $\sigma_{6}$ & $\sigma_{7}$ & $\sigma_{8}$ & $\sigma_{9}$ \\
$\sigma_{9}$ & $\sigma_{8}$ & $\sigma_{6}$ & $\sigma_{7}$ & $\sigma_{8}$ & $\sigma_{9}$ & $\sigma_{8}$ & $\sigma_{9}$ & $\sigma_{6}$ & $\sigma_{7}$
\end{tabular}

If a space $X$ is extremally disconnected, then the closures of open sets are open; compare [2, page 452]. It follows that $\sigma_{6}=\sigma_{12}$. The space $X=\{a, b\}$ with the open sets $X, \emptyset$, and $\{a\}$ is extremally disconnected. But it contains a oneelement open and dense set $\{a\}$, and it follows that $\sigma_{7}=\sigma_{8}$. Similar is for the space $\beta N$; see [2, pages 228 and 453 ] to find the definition and properties of $\beta N$. There are Hausdorff extremally disconnected spaces which are dense in itself. For example, the Stone space of the complete Boolean algebra of all regular closed subsets of the unit interval, compare [12]. For such spaces $\sigma_{7} \neq \sigma_{8}$ and $\sigma_{7}=\sigma_{13}$. To see this, suppose a Hausdorff $X$ is extremally disconnected and dense in itself. Let $X=U \cup V \cup W$, where sets $U, V$, and $W$ are closed and open. Consider a set $A=A^{c-c} \cup B \cup C$, such that

(i) $C^{c-c}=\emptyset$ and $C^{-}=W$;

(ii) $\emptyset \neq B \subseteq V$ and $B^{-c-c}=\emptyset$;

(iii) $U=A^{c-c-} \neq A^{c-c}$.

Then check that

(i) $\sigma_{0}(A)=A$ and $\sigma_{1}(A)=X \backslash\left(A^{c-c} \cup B \cup C\right)$;

(ii) $\sigma_{2}(A)=U \cup B^{-} \cup W$ and $\sigma_{3}(A)=X \backslash A^{c-c}$;

(iii) $\sigma_{4}(A)=V \backslash B^{-}$and $\sigma_{5}(A)=A^{c-c}$;

(iv) $\sigma_{6}(A)=\sigma_{12}(A)=V$ and $\sigma_{7}(A)=\sigma_{13}(A)=U$;

(v) $\sigma_{8}(A)=\sigma_{10}(A)=U \cup W$ and $\sigma_{9}(A)=\sigma_{11}(A)=$ $V \cup W$

Hence we have that $\sigma_{7} \neq \sigma_{8}$. Note that, if $W=\emptyset$, then $\sigma_{7}(A)=$ $\sigma_{8}(A)$. This is the case of subsets of $\beta N$. 


\section{References}

[1] K. Kuratowski, "Sur l'opération $\bar{A}$ de l'analysis situs," Fundamenta Mathematicae, vol. 3, pp. 182-199, 1922.

[2] R. Engelking, General Topology, PWN-Polish Scientific Publishers, Warsaw, Poland, 1977.

[3] T. A. Chapman, "A further note on closure and interior operators," The American Mathematical Monthly, vol. 69, no. 6, pp. 524-529, 1962.

[4] D. Sherman, "Variations on Kuratowski's 14-set theorem," American Mathematical Monthly, vol. 117, no. 2, pp. 113-123, 2010.

[5] B. J. Gardner and M. Jackson, "The Kuratowski closurecomplement theorem," New Zealand Journal of Mathematics, vol. 38, pp. 9-44, 2008.

[6] W. Koenen, "The Kuratowski closure problem in the topology of convexity," The American Mathematical Monthly, vol. 73, pp. 704-708, 1966.

[7] K.-P. Shum, "Closure functions on the set of positive integers," Science in China. Series A, vol. 39, no. 4, pp. 337-346, 1996.

[8] W. Sierpiński, General Topology, Mathematical Expositions, University of Toronto Press, Toronto, Canada, 1956.

[9] A. Cayley, "On the theory of groups," American Journal of Mathematics, vol. 11, no. 2, pp. 139-157, 1889.

[10] C. E. Aull, "Classification of topological spaces," Bulletin de l'Académie Polonaise des Sciences. Série des Sciences Mathématiques, Astronomiques et Physiques, vol. 15, pp. 773-778, 1967.

[11] C. E. Aull, "Corrigendumml: "Classification of topological spaces'”' Bulletin de l'Académie Polonaise des Sciences. Série des Sciences Mathématiques, Astronomiques et Physiques, vol. 16, no. 6, 1968.

[12] A. M. Gleason, "Projective topological spaces," Illinois Journal of Mathematics, vol. 2, pp. 482-489, 1958. 


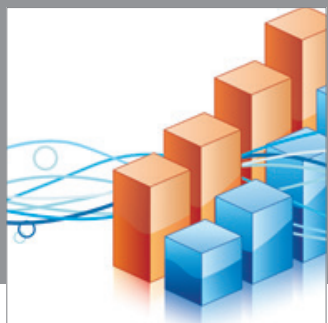

Advances in

Operations Research

mansans

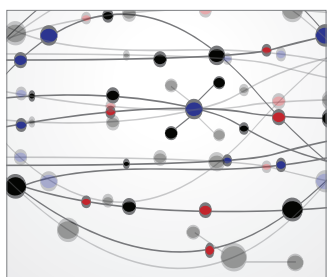

The Scientific World Journal
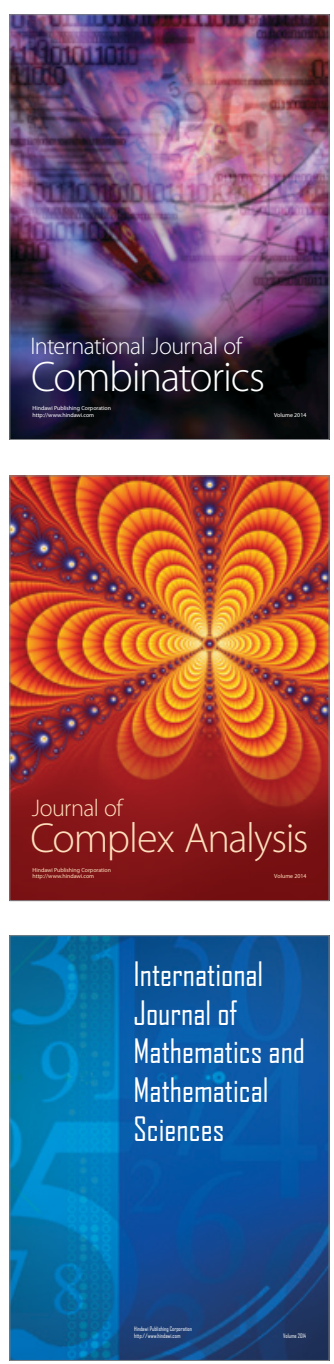
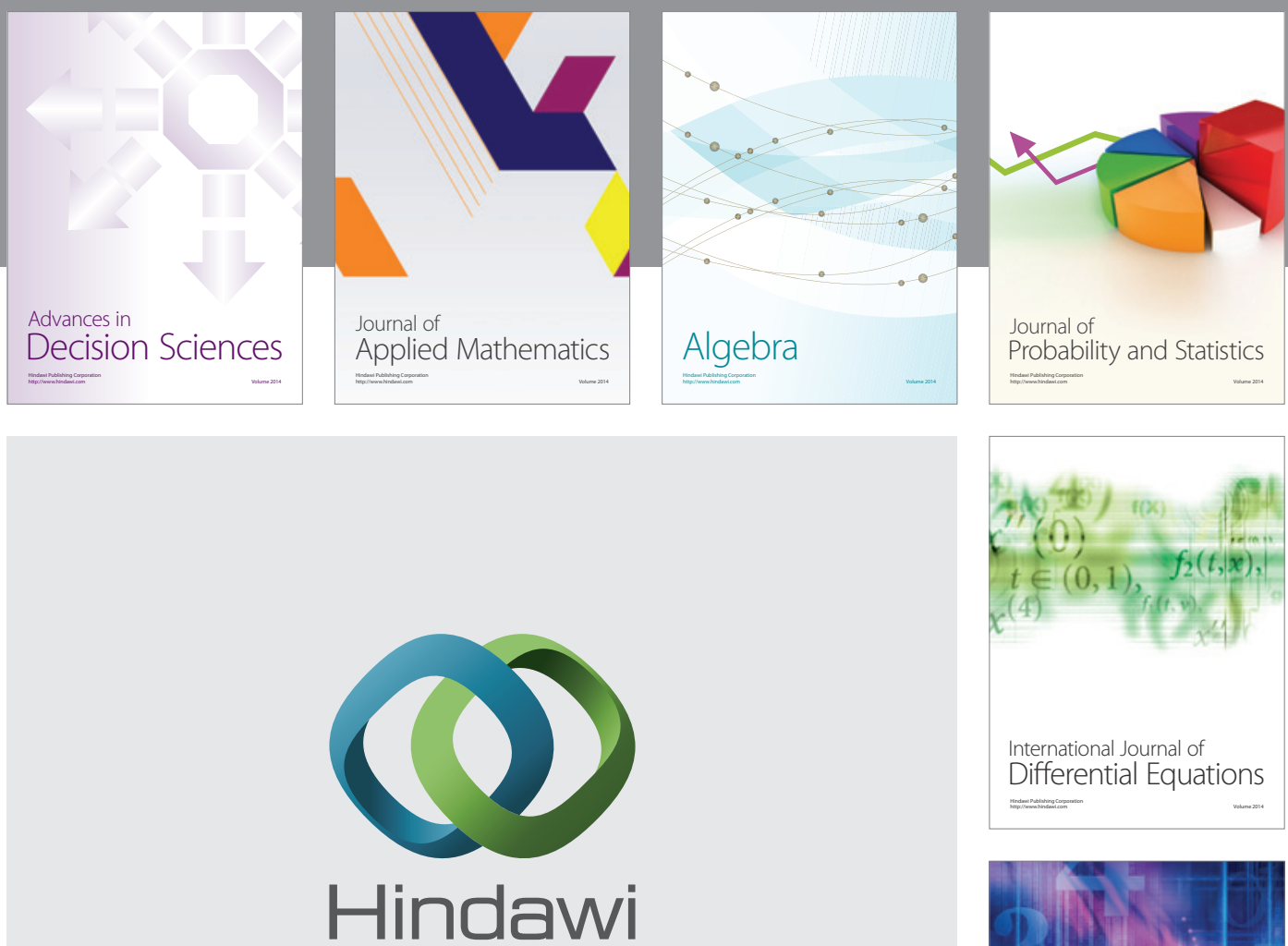

Submit your manuscripts at http://www.hindawi.com
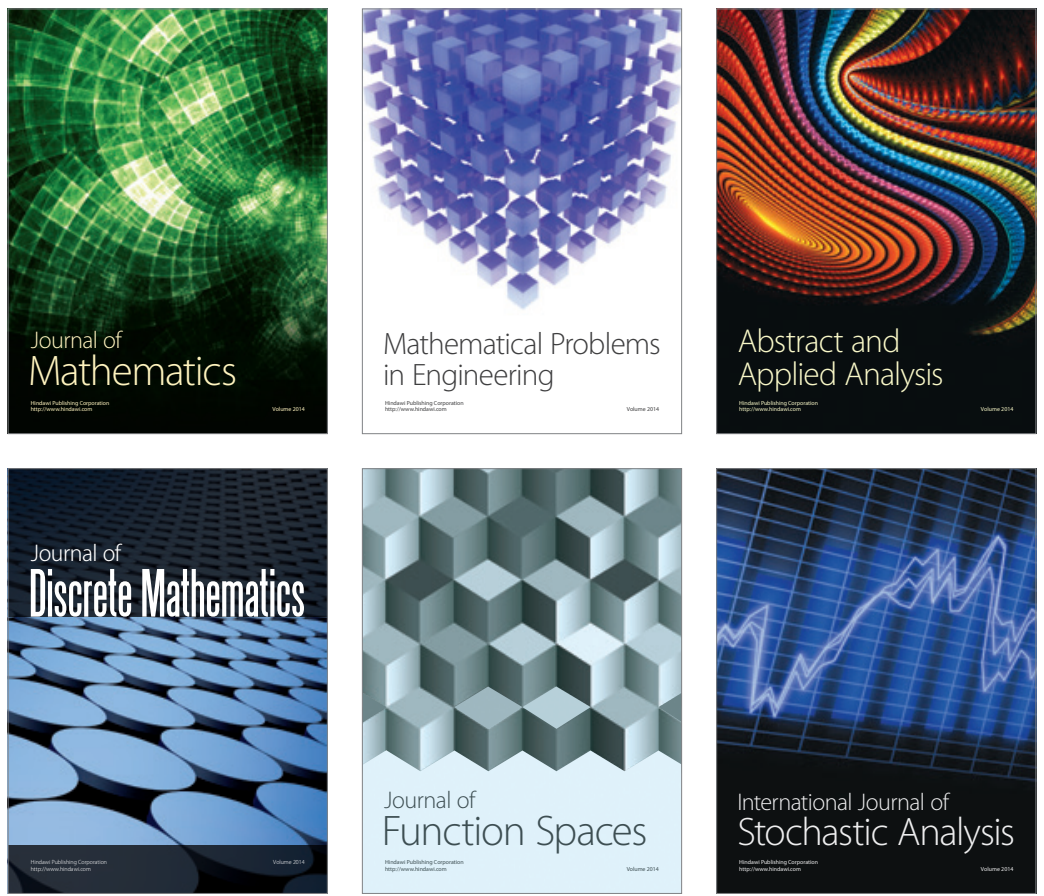

Journal of

Function Spaces

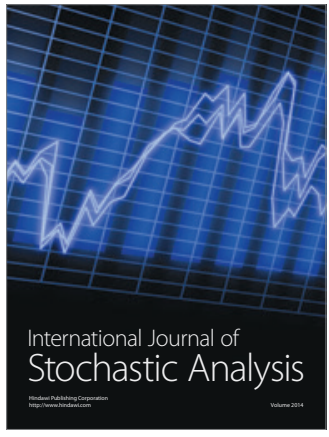

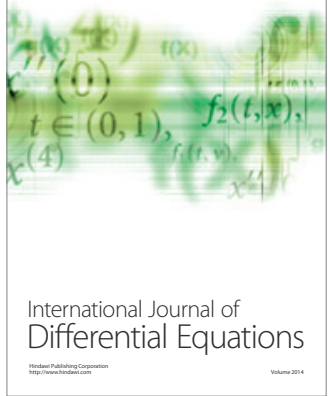
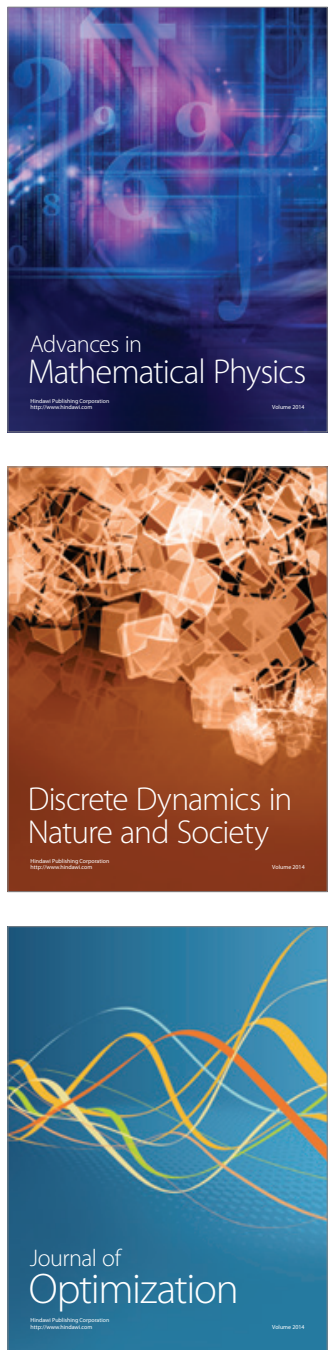